\title{
Integral equation and simulation studies of a planar nematogenic liquid in crossed external fields
}

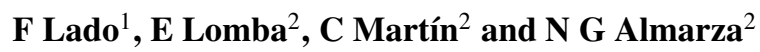 \\ ${ }^{1}$ Department of Physics, North Carolina State University, Raleigh, NC 27695-8202, USA \\ 2 Instituto de Química Física Rocasolano, CSIC, Serrano 119, E-28006 Madrid, Spain
}

Received 14 January 2005, in final form 17 March 2005

Published

Online at stacks.iop.org/JPhysCM/17/1

\begin{abstract}
We study a fluid of nematogenic molecules with centres of mass constrained to lie in a plane but with axes free to rotate in any direction. An external disorienting field perpendicular to the plane along with a second orienting field in the plane induce an in-plane order-disorder transition. We analyse the behaviour of this simple biaxial model using a well-established generalization of molecular integral equation methods built upon specially tailored basis functions that maintain orthogonality in the presence of anisotropy. Computer simulation and integral equation calculations predict an isotropic-nematic transition at low temperatures in zero field and an in-plane transition at somewhat higher temperatures in the presence of the disorienting field. The oriented states obtained in the presence of both fields can subsequently be used as input to uncover in detail first the transition in the absence of the inplane orienting field and finally the spontaneous transition in the absence of any field. According to the simulation, the transition apparently belongs to the Berezinskii-Kosterlitz-Thouless defect-mediated type, whereas the theory reproduces a weak first-order transition.
\end{abstract}

\section{Introduction}

Axial molecules that interact through a pair potential that favours parallel alignment of their axes will, at sufficiently low temperature or high density, spontaneously go over from an isotropic to an orientationally ordered nematic state. This is a uniaxial state, with a preferred nematic direction indicated by a director $\hat{\boldsymbol{n}}$. In the absence of an external orienting field, however, $\hat{\boldsymbol{n}}$ itself may be in any direction and hence a calculated one-body orientational distribution function $f(\omega)$ will still average to a constant. If now an external wall or other disorienting external field $\boldsymbol{W}_{0}$ is brought in that tends to align the molecular axes perpendicular to this field, then the isotropic-nematic transition when it occurs will produce an orientationally ordered state having two relevant directions, those of $\boldsymbol{W}_{0}$ and $\hat{\boldsymbol{n}}$. Again, in the absence of an external ordering field, $\hat{\boldsymbol{n}}$ in this biaxial system may still be in any direction perpendicular to $\boldsymbol{W}_{0}$, and so the calculated $f(\omega)$ will describe only a uniaxial system. To bring out the full

Processing

JPC/cm193055/PAP

Printed 20/4/2005

Focal Image

\begin{tabular}{ll} 
& CRC data \\
\hline File name & First page \\
Date req. & Last page \\
Issue no. & Total pages \\
Artnum & Cover date
\end{tabular}


biaxial nature of this system a second, orienting external field-call it $\boldsymbol{A}_{0}$ - perpendicular to $\boldsymbol{W}_{0}$ must be turned on. Once $\boldsymbol{A}_{0}$ has established a unique direction for $\hat{\boldsymbol{n}}$, this second field may be turned off at some sufficiently low temperature and, with density fixed, the system will remain in the same ordered state as the temperature is raised until a limiting transition temperature is reached when it will revert to a disordered uniaxial state (i.e., with molecular axes on average perpendicular to $\boldsymbol{W}_{0}$ but isotropic in that plane). Further, if both $\boldsymbol{A}_{0}$ and $\boldsymbol{W}_{0}$ are turned off at some ordered low-temperature state, then the field-free system will again remain in the ordered state as the temperature is raised until the order-disorder transition occurs, but now at a lower transition temperature than in the presence of the disorienting field $\boldsymbol{W}_{0}$.

At first sight, one might think it difficult to find a physical interpretation to the disorienting field as introduced above. However, this is actually the simplest model that can account for the physical properties of certain polar molecules in which the dipole moment does not lie along the main symmetry axis, such as para-azoxy-anisole (PAA) [1]. In this instance, the disorienting field accounts for the effect of an external electric field that, acting on the dipole moment, would tend to orient the molecular axis in a direction perpendicular to the field itself. (In the particular case of PAA the angle is actually $62^{\circ}$ rather than $90^{\circ}$.) It also might give a rough representation of complex interactions such as those appearing in paradimethylbenzene near the liquid-vapour interface, where it has been found that the surface effects induce the formation of a planar nematic phase on the interface [2]. Obviously, no physical interpretation of the second disorienting field, $\boldsymbol{A}_{0}$, is needed, since this is just an auxiliary magnitude introduced so as to define a privileged direction when $A_{0} \rightarrow 0$ and thus correctly account for the spontaneously broken symmetry, following the idea of Bogoliubov's quasiaverages [3].

A simple biaxial model exhibiting these properties was recently proposed and studied by Sokolovska, Sokolovskii, and Holovko in three dimensions, with the system in the presence of both an infinite [4] and a finite [5] disorienting field $\boldsymbol{W}_{0}$. The interparticle potential used by these authors corresponds to the Maier-Saupe model for nematogens [6], whose lattice version is known as the Lebwohl-Lasher model [7]. In [4, 5], Sokolovska and co-workers used the anisotropic Ornstein-Zernike equation [8, 9] for the two-body distribution functions along with the exact Lovett-Mou-Buff-Wertheim relation [10, 11] to couple the one-body and two-body functions. A tour de force analytic solution using the mean spherical approximation (MSA) for the needed closure relation allowed them to draw broad conclusions regarding the model's properties, including the transition temperature effect noted above. More recently, the present authors [12] applied the same model interactions to a nematic system with molecular centres constrained to lie in a plane (i.e., a continuous version of the $\mathrm{RP}^{2}$ lattice model studied by Kunz and Zumbach [13]), with $\boldsymbol{W}_{0}$ perpendicular to this plane. That study also used the anisotropic Ornstein-Zernike equation and a different but also exact relation between the one-body and two-body distribution functions: the first member of the Kirkwood-BornGreen-Yvon (KBGY) hierarchy [8]. The approximate closure relation used was the reference hypernetted chain (RHNC) equation [14] and the calculations were performed numerically. (We note that no analytic solution for the MSA is known in two dimensions.) Since the external field destroys the orthogonality of the usual spherical harmonics used in such molecular calculations, a key ingredient of the numerical algorithm was the explicit construction of tailored Legendre functions $\mathcal{P}_{l m}(\cos \theta)$ that maintain orthogonality in the presence of the field $\boldsymbol{W}_{0}$. This approach has been successfully used to describe the ferromagnetic transition in bulk Heisenberg spin fluids [15, 16] as well as the behaviour of a planar dipolar system [17], both in external magnetic fields.

In this paper we extend the two-dimensional calculation of [12] (and partially amend itthe unfortunate use of reduced quantities in the very formulation of this earlier calculation led 
us to inadvertently neglect the temperature dependence in the Boltzmann factor of the external field) by explicitly introducing the second, orienting external field $\boldsymbol{A}_{0}$ in the plane of the system. Following the same prescription as above for the new anisotropy, we build tailored exponential functions $\mathcal{E}_{m}(\cos \phi)$ that maintain orthogonality in the presence of the field $\boldsymbol{A}_{0}$. The overall construction thus yields generalized spherical harmonics $\mathcal{Y}_{l m}(\omega)$ that allow the numerical solution of the integral equation for the biaxial case to proceed with no more complication than that of an isotropic molecular system. As in [12], we use the RHNC closure, which is known to produce relatively accurate results. Having calculated solutions for the biaxially ordered system $\left(W_{0} \neq 0, A_{0} \neq 0\right)$ at low temperatures, we find however that the RHNC closure is not suited for the program described earlier: if the field $\boldsymbol{A}_{0}$ is now turned off, RHNC does not uncover the spontaneous phase transitions expected but rather produces no converged solution at all. Since the MSA is known to have such solutions in three dimensions [4, 5], we turn to a demonstration-of-principle calculation by solving the MSA numerically in two dimensions, using the same algorithm as for RHNC. The expected behaviour is then indeed found. With $A_{0}=0$ and starting from an ordered solution at low temperature, nematic-isotropic transitions are seen as the temperature is raised, with the transition temperature in the presence of the disorienting field $\boldsymbol{W}_{0}$ higher than that of the field-free case. Where comparisons with Monte Carlo (MC) simulations are possible, we find that the MSA closure exacts for this performance a price in accuracy.

One must bear in mind, however, that the ordered states exhibited by this system when $A_{0}=0$-as the simulation will show and as has also been found in lattice model calculations [13, 18]—are of the Berezinskii-Kosterlitz-Thouless (BKT) type [19, 20], i.e., lack proper long-range order, whereas by construction the type of integral equation we use here leads to truly ordered states giving rise to first-order or second-order transitions depending on the magnitude of the disorienting field [4]. This discrepancy as regards the nature of the transition will certainly condition the comparisons between theory and simulation, but we will see that the theory even at the simple MSA level is still able to capture important features of the order-disorder transition.

\section{Crossed external fields}

The total potential energy $U$ of the $N$-molecule system in a given configuration is

$$
U=\sum_{j} u_{z}\left(\omega_{j}\right)+\sum_{j} u_{x}\left(\omega_{j}\right)+\sum_{i<j} u_{\mathrm{HS}}\left(r_{i j}\right)+\sum_{i<j} u\left(r_{i j}, \omega_{i}, \omega_{j}\right),
$$

where $\omega=(\theta, \phi)$ gives the orientation of a molecular nematic axis, which is free to rotate in three dimensions, referred to the $z$ axis perpendicular to the system plane of area $A$. The energies here are the external disorienting potential in the $z$ direction,

$$
u_{z}(\omega)=W_{0} P_{2}(\cos \theta), \quad \text { with } W_{0} \geqslant 0,
$$

which tends to rotate the molecular axes into the $x y$ system plane, the external orienting potential,

$$
u_{x}(\omega)=-A_{0} T_{2}(\cos \phi), \quad \text { with } A_{0} \geqslant 0,
$$

which tends to align the molecular axes along an in-plane direction (denoted as the $x$ axis), the hard sphere potential, $u_{\mathrm{HS}}(r)$, for spheres of diameter $\sigma$, and an attractive potential between a pair of molecules that tends to align their mutual nematic axes,

$$
\begin{aligned}
& u\left(r, \omega_{1}, \omega_{2}\right)=-K u_{0}(r) P_{2}\left(\cos \theta_{12}\right), \quad \text { with } K>0, \\
& u_{0}(r)=\frac{\mathrm{e}^{-\kappa(r-\sigma)}}{r / \sigma}, \quad \text { for } r>\sigma .
\end{aligned}
$$


In these expressions, $P_{2}(x)=\left(3 x^{2}-1\right) / 2$ is the Legendre polynomial of order two and $T_{2}(x)=2 x^{2}-1$ the Chebyshev polynomial of order two, while $\theta_{12}$ is the angle between the axes of molecules 1 and 2 and $\kappa$ is a range parameter.

In the calculation, the dimensionless inverse coupling strength $(\beta K)^{-1}=k_{\mathrm{B}} T / K \equiv T^{*}$, where $T$ is the absolute temperature and $k_{\mathrm{B}}$ is Boltzmann's constant, will serve to define the reduced temperature $T^{*}$. The total potential energy in units of $k_{\mathrm{B}} T$ is then

$$
\begin{aligned}
\frac{U}{k_{\mathrm{B}} T}=\frac{1}{T^{*}}[ & \left.\frac{W_{0}}{K} \sum_{j} P_{2}\left(\cos \theta_{j}\right)-\frac{A_{0}}{K} \sum_{j} T_{2}\left(\cos \phi_{j}\right)-\sum_{i<j} u_{0}\left(r_{i j}\right) P_{2}\left(\cos \theta_{i j}\right)\right] \\
& +\sum_{i<j} \beta u_{\mathrm{HS}}\left(r_{i j}\right) .
\end{aligned}
$$

We introduce the one-body distribution functions for noninteracting molecules in the external fields, $f_{0 z}(\cos \theta)$ and $f_{0 x}(\cos \phi)$, by factoring the canonical partition function

$$
Z=\frac{1}{N !\left(4 \pi \Lambda^{2}\right)^{N}} \int \prod_{j=1}^{N}\left(\mathrm{~d} \boldsymbol{r}_{j} \mathrm{~d} \omega_{j}\right) \mathrm{e}^{-\beta U}
$$

into an ideal part and the excess, $Z=Z^{\text {id }} Z^{\text {ex }}$, where

$$
\begin{aligned}
Z^{\text {id }} & =\frac{1}{N !\left(4 \pi \Lambda^{2}\right)^{N}} \int \prod_{j=1}^{N}\left(\mathrm{~d} r_{j} \mathrm{~d} \omega_{j}\right) \exp \left(-\beta \sum_{j}\left[u_{z}\left(\omega_{j}\right)+u_{x}\left(\omega_{j}\right)\right]\right) \\
& =\frac{1}{N !}\left(\frac{A}{\Lambda^{2}} \frac{\operatorname{erf}\left[\left(3 \beta W_{0} / 2\right)^{1 / 2}\right] \mathrm{e}^{\beta W_{0} / 2}}{2\left(3 \beta W_{0} / 2 \pi\right)^{1 / 2}} I_{0}\left(\beta A_{0}\right)\right)^{N}
\end{aligned}
$$

and so

$Z^{\mathrm{ex}}=\frac{1}{(4 \pi A)^{N}} \int \prod_{j=1}^{N}\left[\mathrm{~d} \boldsymbol{r}_{j} \mathrm{~d} \omega_{j} f_{0}\left(\omega_{j}\right)\right] \exp \left(-\beta \sum_{i<j}\left[u_{\mathrm{HS}}\left(r_{i j}\right)+u\left(r_{i j}, \omega_{i}, \omega_{j}\right)\right]\right)$.

Here we have put $f_{0}(\omega) \equiv f_{0 z}(\cos \theta) f_{0 x}(\cos \phi)$, with

$$
\begin{aligned}
f_{0 z}(\cos \theta) & =\frac{2\left(3 \beta W_{0} / 2 \pi\right)^{1 / 2}}{\operatorname{erf}\left[\left(3 \beta W_{0} / 2\right)^{1 / 2}\right]} \exp \left(-\frac{3}{2} \beta W_{0} \cos ^{2} \theta\right), \\
f_{0 x}(\cos \phi) & =\frac{\exp \left(\beta A_{0} \cos 2 \phi\right)}{I_{0}\left(\beta A_{0}\right)},
\end{aligned}
$$

where $\operatorname{erf}(x)$ is the error function and $I_{0}(x)$ the modified Bessel function of order zero. We have further conventionally introduced above the de Broglie thermal wavelength $\Lambda$ for the normalization of $Z$. The distribution function $f_{0}(\omega)$ is normalized so that

$$
\frac{1}{4 \pi} \int \mathrm{d} \omega f_{0}(\omega)=1
$$

or individually,

$$
\begin{aligned}
& \frac{1}{2} \int_{0}^{\pi} \mathrm{d} \theta \sin \theta f_{0 z}(\cos \theta)=1 \\
& \frac{1}{2 \pi} \int_{0}^{2 \pi} \mathrm{d} \phi f_{0 x}(\cos \phi)=1
\end{aligned}
$$




\section{Formulation of the integral equations}

\subsection{One-body and two-body distribution functions}

With the molecules subject to both one-body and two-body interactions, a statistical description of the system requires both the one-body and two-body density functions,

$$
\begin{gathered}
\rho^{(1)}(\boldsymbol{r}, \omega)=\left\langle\sum_{j=1}^{N} \delta\left(\boldsymbol{r}-\boldsymbol{r}_{j}\right) \delta\left(\omega-\omega_{j}\right)\right\rangle=\frac{\rho}{4 \pi} f(\omega), \\
\rho^{(2)}\left(\boldsymbol{r}, \omega, \boldsymbol{r}^{\prime}, \omega^{\prime}\right)=\left\langle\sum_{i \neq j} \delta\left(\boldsymbol{r}-\boldsymbol{r}_{i}\right) \delta\left(\omega-\omega_{i}\right) \delta\left(\boldsymbol{r}^{\prime}-\boldsymbol{r}_{j}\right) \delta\left(\omega^{\prime}-\omega_{j}\right)\right\rangle \\
=\frac{\rho^{2}}{(4 \pi)^{2}} f(\omega) f\left(\omega^{\prime}\right) g\left(\left|\boldsymbol{r}-\boldsymbol{r}^{\prime}\right|, \omega, \omega^{\prime}\right),
\end{gathered}
$$

where $\rho=N / A$ is the planar density and $f(\omega)$ the one-body angular distribution function in the interacting fluid. Equation (16) defines the generalized pair distribution function $g\left(r, \omega, \omega^{\prime}\right)$ of the anisotropic system. The angular brackets in these definitions denote a canonical ensemble average with the potential of equation (1).

The basic equations that determine the distribution functions $f(\omega)$ and $g\left(r, \omega, \omega^{\prime}\right)$ are well known $[8,9]$. The one-body density can be differentiated with respect to $\theta$ to give

$\frac{\mathrm{d}}{\mathrm{d} \theta_{1}} \ln \left[\frac{f\left(\omega_{1}\right)}{f_{0}\left(\omega_{1}\right)}\right]=-\frac{\rho}{4 \pi} \int \mathrm{d} \boldsymbol{r}_{2} \mathrm{~d} \omega_{2} f\left(\omega_{2}\right) g\left(r_{12}, \omega_{1}, \omega_{2}\right) \frac{\mathrm{d}}{\mathrm{d} \theta_{1}} \beta u\left(r_{12}, \omega_{1}, \omega_{2}\right)$,

which will determine $f_{z}(\cos \theta)$, and with respect to $\phi$ to give

$\frac{\mathrm{d}}{\mathrm{d} \phi_{1}} \ln \left[\frac{f\left(\omega_{1}\right)}{f_{0}\left(\omega_{1}\right)}\right]=-\frac{\rho}{4 \pi} \int \mathrm{d} \boldsymbol{r}_{2} \mathrm{~d} \omega_{2} f\left(\omega_{2}\right) g\left(r_{12}, \omega_{1}, \omega_{2}\right) \frac{\mathrm{d}}{\mathrm{d} \phi_{1}} \beta u\left(r_{12}, \omega_{1}, \omega_{2}\right)$,

which will determine $f_{x}(\cos \phi)$. These are first-order members of the KBGY hierarchy. Calculation of $f(\omega)=f_{z}(\cos \theta) f_{x}(\cos \phi)$ from these equations requires knowing $g\left(r, \omega_{1}, \omega_{2}\right)$. In classical liquid state theory, the pair distribution function is obtained from the OrnsteinZernike (OZ) equation and a closure relation [8,9]. The first of these, generalized for anisotropy, reads

$\gamma\left(r_{12}, \omega_{1}, \omega_{2}\right)=\frac{\rho}{4 \pi} \int \mathrm{d} r_{3} \mathrm{~d} \omega_{3} f\left(\omega_{3}\right)\left[\gamma\left(r_{13}, \omega_{1}, \omega_{3}\right)+c\left(r_{13}, \omega_{1}, \omega_{3}\right)\right] c\left(r_{32}, \omega_{3}, \omega_{2}\right)$

for the indirect correlation function $\gamma=g-1-c$, where $c\left(r, \omega_{1}, \omega_{2}\right)$ is the direct correlation function. The second, or closure, relation expresses $c\left(r, \omega_{1}, \omega_{2}\right)$ back in terms of $\gamma\left(r, \omega_{1}, \omega_{2}\right)$ and the model's pair interactions,

$$
\begin{aligned}
c\left(r, \omega_{1}, \omega_{2}\right)= & \exp \left[-\beta u_{\mathrm{HS}}(r)-\beta u\left(r, \omega_{1}, \omega_{2}\right)+\gamma\left(r, \omega_{1}, \omega_{2}\right)\right. \\
& \left.+b\left(r, \omega_{1}, \omega_{2}\right)\right]-1-\gamma\left(r, \omega_{1}, \omega_{2}\right) .
\end{aligned}
$$

This relation must be supplemented with an approximation for $b\left(r, \omega_{1}, \omega_{2}\right)$, the so-called bridge function, which is formally defined in terms of a diagram summation [8] that offers little practical benefit. Most approximate closures for $c\left(r, \omega_{1}, \omega_{2}\right)$ define $b\left(r, \omega_{1}, \omega_{2}\right)$ implicitly.

\subsection{Generalized spherical harmonics}

The usual spherical harmonic expansion for a pair function, say $g\left(r, \omega_{1}, \omega_{2}\right)$, may be written as 


$$
\begin{aligned}
g\left(r, \omega_{1}, \omega_{2}\right)= & 4 \pi \sum_{l_{1}, l_{2}, m_{1}, m_{2}} G_{l_{1} l_{2}}^{m_{1} m_{2}}(r) Y_{l_{1} m_{1}}\left(\omega_{1}\right) Y_{l_{2} \bar{m}_{2}}\left(\omega_{2}\right) \\
= & \sum_{l_{1}, l_{2}, m_{1}, m_{2}}(-1)^{m_{1}} G_{l_{1} l_{2}}^{m_{1} m_{2}}(r) \hat{P}_{l_{1} m_{1}}\left(\cos \theta_{1}\right) \hat{P}_{l_{2} m_{2}}\left(\cos \theta_{2}\right) \\
& \times E_{m_{1}}\left(\cos \phi_{1}\right) E_{m_{2}}^{*}\left(\cos \phi_{2}\right),
\end{aligned}
$$

where $\bar{m} \equiv-m$, asterisk denotes complex conjugate, and

$$
\begin{aligned}
E_{m}(\cos \phi) & \equiv \mathrm{e}^{\mathrm{i} m \phi} \\
& =T_{m}(\cos \phi)+\mathrm{i} V_{m}(\cos \phi)
\end{aligned}
$$

Here $T_{m}(\cos \phi)=\cos m \phi$ is the Chebyshev polynomial of order $m$ and $V_{m}(\cos \phi)=\sin m \phi$ the associated Chebyshev function of order $m$. In the second equality of equation (21), we are using Legendre functions $\hat{P}_{l m}(x)$ that are normalized such that

$$
\frac{1}{2} \int_{0}^{\pi} \mathrm{d} \theta \sin \theta \hat{P}_{l m}(\cos \theta) \hat{P}_{l^{\prime} m}(\cos \theta)=\delta_{l l^{\prime}} .
$$

The exponential functions $E_{m}(\cos \phi)$ of course have the normalization

$$
\frac{1}{2 \pi} \int_{0}^{2 \pi} \mathrm{d} \phi E_{m}(\cos \phi) E_{m^{\prime}}^{*}(\cos \phi)=\delta_{m m^{\prime}}
$$

for $m, m^{\prime}$ positive or negative. We write the expansion coefficients in equation (21) in upper case, $G$, to distinguish them from the distinct set of coefficients written in lower case, $g$, to be introduced next.

The external fields along the $z$ and $x$ axes produce nonuniform distributions $f_{z}(\cos \theta)$ and $f_{x}(\cos \phi)$ that destroy the orthogonality of the Legendre functions and exponential functions, respectively. To recover orthogonality and work conveniently within these external fields, we generalize equation (21) to read

$$
\begin{aligned}
g\left(r, \omega_{1}, \omega_{2}\right)= & 4 \pi \sum_{l_{1}, l_{2}, m_{1}, m_{2}} g_{l_{1} l_{2}}^{m_{1} m_{2}}(r) \mathcal{Y}_{l_{1} m_{1}}\left(\omega_{1}\right) \mathcal{Y}_{l_{2} \bar{m}_{2}}\left(\omega_{2}\right) \\
= & \sum_{l_{1}, l_{2}, m_{1}, m_{2}}(-1)^{m_{1}} g_{l_{1} l_{2}}^{m_{1} m_{2}}(r) \mathcal{P}_{l_{1} m_{1}}\left(\cos \theta_{1}\right) \mathcal{P}_{l_{2} m_{2}}\left(\cos \theta_{2}\right) \\
& \times \mathcal{E}_{m_{1}}\left(\cos \phi_{1}\right) \mathcal{E}_{m_{2}}^{*}\left(\cos \phi_{2}\right),
\end{aligned}
$$

and construct new basis functions $\mathcal{P}_{l m}(\cos \theta)$ and $\mathcal{E}_{m}(\cos \phi)$ with the orthonormalizations

$$
\frac{1}{2} \int_{0}^{\pi} \mathrm{d} \theta \sin \theta f_{z}(\cos \theta) \mathcal{P}_{l m}(\cos \theta) \mathcal{P}_{l^{\prime} m}(\cos \theta)=\delta_{l l^{\prime}}
$$

for the generalized Legendre functions and

$$
\frac{1}{2 \pi} \int_{0}^{2 \pi} \mathrm{d} \phi f_{x}(\cos \phi) \mathcal{E}_{m}(\cos \phi) \mathcal{E}_{m^{\prime}}^{*}(\cos \phi)=\delta_{m m^{\prime}}
$$

again for $m, m^{\prime}$ positive or negative, for the generalized exponential functions. More specifically, for the latter we have

$$
\mathcal{E}_{m}(\cos \phi)=\mathcal{T}_{m}(\cos \phi)+\mathrm{i} \mathcal{V}_{m}(\cos \phi),
$$

where the generalized Chebyshev functions satisfy 
$\frac{1}{2 \pi} \int_{0}^{2 \pi} \mathrm{d} \phi f_{x}(\cos \phi) \mathcal{T}_{m}(\cos \phi) \mathcal{T}_{m^{\prime}}(\cos \phi)= \begin{cases}1, & m=m^{\prime}=0 \\ 1 / 2, & m=m^{\prime}>0 \\ 0, & m \neq m^{\prime}\end{cases}$
$\frac{1}{2 \pi} \int_{0}^{2 \pi} \mathrm{d} \phi f_{x}(\cos \phi) \mathcal{V}_{m}(\cos \phi) \mathcal{V}_{m^{\prime}}(\cos \phi)= \begin{cases}0, & m=m^{\prime}=0 \\ 1 / 2, & m=m^{\prime}>0 \\ 0, & m \neq m^{\prime}\end{cases}$

$\frac{1}{2 \pi} \int_{0}^{2 \pi} \mathrm{d} \phi f_{x}(\cos \phi) \mathcal{T}_{m}(\cos \phi) \mathcal{V}_{m^{\prime}}(\cos \phi)=0$,

for $m, m^{\prime} \geqslant 0$. The generalized spherical harmonics introduced above follow the CondonShortley phase convention usually chosen for the standard set [21], which results in the definitions

$$
\begin{aligned}
& \mathcal{Y}_{l m}(\omega)=\frac{(-1)^{m}}{\sqrt{4 \pi}} \mathcal{P}_{l m}(\cos \theta) \mathcal{E}_{m}(\cos \phi), \\
& \mathcal{Y}_{l \bar{m}}(\omega)=\frac{1}{\sqrt{4 \pi}} \mathcal{P}_{l m}(\cos \theta) \mathcal{E}_{m}^{*}(\cos \phi)
\end{aligned}
$$

for $m \geqslant 0$. They are orthonormal with weight function $f(\omega)=f_{z}(\cos \theta) f_{x}(\cos \phi)$,

$$
\int \mathrm{d} \omega f(\omega) \mathcal{Y}_{l m}(\omega) \mathcal{Y}_{l^{\prime} m^{\prime}}^{*}(\omega)=\delta_{l l^{\prime}} \delta_{m m^{\prime}}
$$

Construction of the generalized Legendre functions has been previously described [17]. The generalized Chebyshev functions needed are given explicitly in the appendix.

Keeping all terms in these expansions up to $l_{1}=l_{2}=\left|m_{1}\right|=\left|m_{2}\right|=$ maxM requires $1,9,39,118, \ldots$ distinct coefficients for $\max M=0,2,4,6, \ldots$ respectively. The results reported below are for maxM $=4$ using 39 distinct coefficients; essentially identical results are obtained with $\operatorname{maxM}=2$ using just nine distinct coefficients.

Applied to the pair potential $u\left(r, \omega_{1}, \omega_{2}\right)$, the expansion in equation (25) becomes

$$
u\left(r, \omega_{1}, \omega_{2}\right)=-4 \pi K u_{0}(r) \sum_{l_{1}, l_{2}, m_{1}, m_{2}} P_{l_{1} l_{2}}^{m_{1} m_{2}} \mathcal{Y}_{l_{1} m_{1}}\left(\omega_{1}\right) \mathcal{Y}_{l_{2} \bar{m}_{2}}\left(\omega_{2}\right),
$$

with nine distinct nonvanishing coefficients in the general case:

$$
\begin{aligned}
& P_{00}^{00}=\frac{1}{4}\left(3 m_{2}-1\right)^{2}+\frac{3}{4} \mu_{2}^{2}\left(1-m_{2}\right)^{2}, \\
& P_{20}^{00}=P_{02}^{00}=\frac{3}{4}\left[\left(3 m_{2}-1\right)-\mu_{2}^{2}\left(1-m_{2}\right)\right]\left(m_{4}-m_{2}^{2}\right)^{1 / 2}, \\
& P_{20}^{20}=P_{02}^{02}=\frac{3}{8} \mu_{2}\left(1-2 \mu_{2}^{2}+\mu_{4}\right)^{1 / 2}\left(1-m_{2}\right)\left(1-2 m_{2}+m_{4}\right)^{1 / 2}, \\
& P_{22}^{00}=\frac{3}{4}\left(3+\mu_{2}^{2}\right)\left(m_{4}-m_{2}^{2}\right), \\
& P_{22}^{20}=P_{22}^{02}=-\frac{3}{8} \mu_{2}\left(1-2 \mu_{2}^{2}+\mu_{4}\right)^{1 / 2}\left(m_{4}-m_{2}^{2}\right)^{1 / 2}\left(1-2 m_{2}+m_{4}\right)^{1 / 2}, \\
& P_{22}^{11}=-\frac{3}{2}\left(m_{2}-m_{4}\right), \\
& P_{22}^{1-1}=-\frac{3}{2} \mu_{2}\left(m_{2}-m_{4}\right), \\
& P_{22}^{22}=\frac{3}{8}\left(1-\mu_{2}^{2}\right)\left(1-2 m_{2}+m_{4}\right), \\
& P_{22}^{2-2}=\frac{3}{8}\left(\mu_{4}-\mu_{2}^{2}\right)\left(1-2 m_{2}+m_{4}\right) .
\end{aligned}
$$

Here $m_{k}=\left\langle(\cos \theta)^{k}\right\rangle$ is the $k$ th moment of $f_{z}(\cos \theta)$ and $\mu_{k}=\langle\cos k \phi\rangle$ the $k$ th pseudomoment of $f_{x}(\cos \phi)$. If the orienting field along the $x$ axis is turned off, then $\mu_{2}=\mu_{4}=0$ and the set reduces to five distinct nonvanishing coefficients [12]; if further the disorienting field along the $z$ axis is also turned off, then $m_{2}=1 / 3, m_{4}=1 / 5$, and just three contributions remain, $P_{22}^{00}=-P_{22}^{11}=P_{22}^{22}=1 / 5$. 


\subsection{Ornstein-Zernike equation}

The OZ equation (19) is more conveniently used after deconvolution by Fourier transformation, which yields

$\tilde{\gamma}\left(k, \omega_{1}, \omega_{2}\right)=\frac{\rho}{4 \pi} \int \mathrm{d} \omega_{3} f\left(\omega_{3}\right)\left[\tilde{\gamma}\left(k, \omega_{1}, \omega_{3}\right)+\tilde{c}\left(k, \omega_{1}, \omega_{3}\right)\right] \tilde{c}\left(k, \omega_{3}, \omega_{2}\right)$,

with a final integration still remaining. Because the orientations of the molecular axes $\omega_{1}, \omega_{2}$ and that of $\boldsymbol{r}_{12}$ are decoupled in the present model, the transforms may be performed holding the former fixed. In two dimensions, the Fourier transform (FT) of a circularly symmetric function becomes a Hankel transform, and so we get a transform pair

$$
\begin{aligned}
& \tilde{\gamma}\left(k, \omega_{1}, \omega_{2}\right)=2 \pi \int_{0}^{\infty} \mathrm{d} r r \gamma\left(r, \omega_{1}, \omega_{2}\right) J_{0}(k r), \\
& \gamma\left(r, \omega_{1}, \omega_{2}\right)=\frac{1}{2 \pi} \int_{0}^{\infty} \mathrm{d} k k \tilde{\gamma}\left(k, \omega_{1}, \omega_{2}\right) J_{0}(k r),
\end{aligned}
$$

where $J_{0}(x)$ is the Bessel function of order zero. The transformed functions are then expanded just as in equation (25),

$$
\tilde{\gamma}\left(k, \omega_{1}, \omega_{2}\right)=4 \pi \sum_{l_{1}, l_{2}, m_{1}, m_{2}} \tilde{\gamma}_{l_{1} l_{2}}^{m_{1} m_{2}}(k) \mathcal{Y}_{l_{1} m_{1}}\left(\omega_{1}\right) \mathcal{Y}_{l_{2} \bar{m}_{2}}\left(\omega_{2}\right)
$$

and similarly for $\tilde{c}\left(k, \omega_{1}, \omega_{2}\right)$, and the final integration in equation (44) is carried out to give a matrix equation,

$$
\tilde{\gamma}_{l_{1} l_{2}}^{m_{1} m_{2}}(k)=\rho \sum_{l_{3}, m_{3}}(-1)^{m_{3}}\left[\tilde{\gamma}_{l_{1} l_{3}}^{m_{1} m_{3}}(k)+\tilde{c}_{l_{1} l_{3}}^{m_{1} m_{3}}(k)\right] \tilde{c}_{l_{3} l_{2}}^{m_{3} m_{2}}(k)
$$

or

$$
\sum_{l_{3}, m_{3}} \tilde{\gamma}_{l_{1} l_{3}}^{m_{1} m_{3}}(k)\left[\delta_{l_{3} l_{2}} \delta_{m_{3} m_{2}}-(-1)^{m_{3}} \rho \tilde{c}_{l_{3} l_{2}}^{m_{3} m_{2}}(k)\right]=\rho \sum_{l_{3}, m_{3}}(-1)^{m_{3}} \tilde{c}_{l_{1} l_{3}}^{m_{1} m_{3}}(k) \tilde{c}_{l_{3} l_{2}}^{m_{3} m_{2}}(k)
$$

which can be solved by matrix manipulations for the $\tilde{\gamma}_{l_{1} l_{2}}^{m_{1} m_{2}}(k)$ in terms of the $\tilde{c}_{l_{1} l_{2}}^{m_{1} m_{2}}(k)$. One cycle of the iterative (numerical) solution that yields a converged set of coefficients $\left\{\gamma_{l_{1} l_{2}}^{m_{1} m_{2}}(r)\right\}$ will then consist of four steps:

$\left\{\gamma_{l_{1} l_{2}}^{m_{1} m_{2}}(r)\right\} \stackrel{\text { closure }}{\longrightarrow}\left\{c_{l_{1} l_{2}}^{m_{1} m_{2}}(r)\right\} \stackrel{(\mathrm{FT})}{\longrightarrow}\left\{\tilde{c}_{l_{1} l_{2}}^{m_{1} m_{2}}(k)\right\} \stackrel{\mathrm{OZ}}{\longrightarrow}\left\{\tilde{\gamma}_{l_{1} l_{2}}^{m_{1} m_{2}}(k)\right\} \stackrel{(\mathrm{FT})^{-1}}{\longrightarrow}\left\{\gamma_{l_{1} l_{2}}^{m_{1} m_{2}}(r)\right\}$

Keeping terms up to order two in the expansions, which captures the essential properties of the system, we note that the coefficients

$$
C_{l_{1} l_{2}}^{m_{1} m_{2}}(k) \equiv \delta_{l_{1} l_{2}} \delta_{m_{1} m_{2}}-(-1)^{m_{1}} \rho \tilde{c}_{l_{1} l_{2}}^{m_{1} m_{2}}(k)
$$

form a symmetric $6 \times 6$ matrix, 


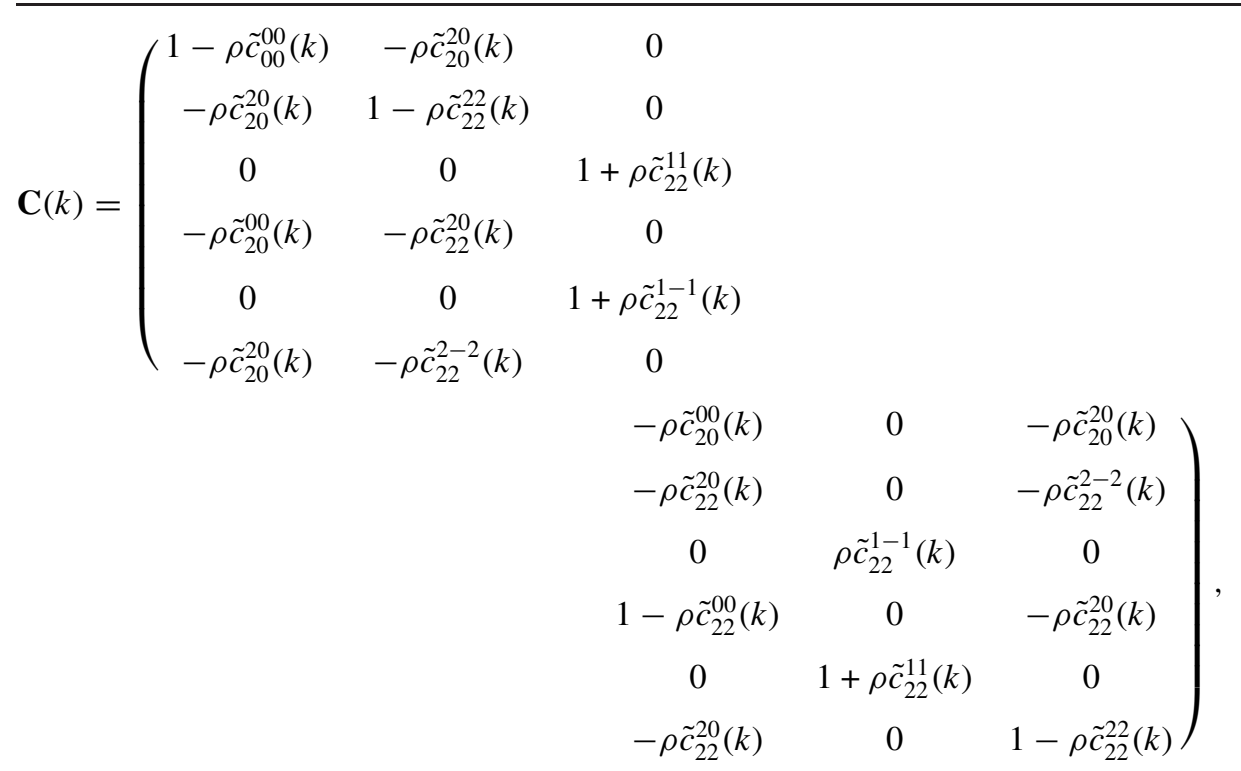

where the symmetries $\tilde{c}_{l_{1} l_{2}}^{m_{1} m_{2}}(k)=\tilde{c}_{l_{1} l_{2}}^{\bar{m}_{1} \bar{m}_{2}}(k)=\tilde{c}_{l_{2} l_{1}}^{m_{2} m_{1}}(k)$ have been used to reduce the number of distinct coefficients to nine. For the general case with $W_{0}>0$ and $A_{0}>0$, all of the transforms displayed above are nonzero. A simple program in Mathematica can factor the characteristic polynomial of this matrix and analytically extract three eigenvalues,

$$
\begin{aligned}
& \lambda_{1}(k)=1+\rho \tilde{c}_{22}^{11}(k)-\rho \tilde{c}_{22}^{1-1}(k), \\
& \lambda_{2}(k)=1+\rho \tilde{c}_{22}^{11}(k)+\rho \tilde{c}_{22}^{1-1}(k), \\
& \lambda_{3}(k)=1-\rho \tilde{c}_{22}^{22}(k)+\rho \tilde{c}_{22}^{2-2}(k) .
\end{aligned}
$$

Similarly, the inverse matrix

$$
\mathbf{H}(k)=[\mathbf{C}(k)]^{-1},
$$

with elements

$$
H_{l_{1} l_{2}}^{m_{1} m_{2}}(k) \equiv \delta_{l_{1} l_{2}} \delta_{m_{1} m_{2}}+(-1)^{m_{1}} \rho \tilde{h}_{l_{1} l_{2}}^{m_{1} m_{2}}(k)
$$

is analytically evaluated to yield

$$
\begin{aligned}
& v_{1}(k) \equiv 1-\rho \tilde{h}_{22}^{11}(k)+\rho \tilde{h}_{22}^{1-1}(k)=\left[1+\rho \tilde{c}_{22}^{11}(k)-\rho \tilde{c}_{22}^{1-1}(k)\right]^{-1}=\lambda_{1}^{-1}(k), \\
& \nu_{2}(k) \equiv 1-\rho \tilde{h}_{22}^{11}(k)-\rho \tilde{h}_{22}^{1-1}(k)=\left[1+\rho \tilde{c}_{22}^{11}(k)+\rho \tilde{c}_{22}^{1-1}(k)\right]^{-1}=\lambda_{2}^{-1}(k), \\
& \nu_{3}(k) \equiv 1+\rho \tilde{h}_{22}^{22}(k)-\rho \tilde{h}_{22}^{2-2}(k)=\left[1-\rho \tilde{c}_{22}^{22}(k)+\rho \tilde{c}_{22}^{2-2}(k)\right]^{-1}=\lambda_{3}^{-1}(k) .
\end{aligned}
$$

It should be noted that these remarkably simple expressions hold only when the specially tailored functions $\mathcal{P}_{l m}(\cos \theta)$ and $\mathcal{E}_{m}(\cos \phi)$ are used as bases for the spherical harmonic expansions. The usual bases of Legendre functions $P_{l m}(\cos \theta)$ and exponentials $E_{m}(\cos \phi)$ would produce hopelessly entangled coefficients.

The Hankel transforms needed in these calculations must be evaluated numerically. The discrete versions $[22,23]$ of the integrals (45) and (46) used in this work,

$$
\begin{aligned}
& \tilde{f}\left(k_{j}\right)=\frac{4 \pi}{K^{2}} \sum_{i=1}^{N_{r}-1} f\left(r_{i}\right) \frac{J_{0}\left(k_{j} r_{i}\right)}{J_{1}^{2}\left(K r_{i}\right)}, \\
& f\left(r_{i}\right)=\frac{1}{\pi R^{2}} \sum_{j=1}^{N_{r}-1} \tilde{f}\left(k_{j}\right) \frac{J_{0}\left(k_{j} r_{i}\right)}{J_{1}^{2}\left(k_{j} R\right)},
\end{aligned}
$$


preserve the orthogonality of the continuous Hankel transforms. In these expressions, $J_{n}(x)$ is the Bessel function of order $n$ and the numerical grids are defined by $r_{i}=x_{i} / K, k_{j}=x_{j} / R$, where $x_{k}$ is the $k$ th root of $J_{0}(x)$ and $K \equiv k_{N_{r}}, R \equiv r_{N_{r}}$; the range $R$ and number of grid points $N_{r}$ are free choices. In the calculations reported below, we have used $R=20 \sigma$ and $N_{r}=1000$.

\subsection{KBGY equations for $f(\omega)=f_{z}(\cos \theta) f_{x}(\cos \phi)$}

The iterative solution for $\gamma\left(r_{12}, \omega_{1}, \omega_{2}\right)$ described in the previous section depends on the current form of the angular distribution function $f(\omega)=f_{z}(\cos \theta) f_{x}(\cos \phi)$, as reflected in the pair potential coefficients $P_{l_{1} l_{2}}^{m_{1} m_{2}}$, equations (35)-(43); now $f(\omega)$ must be updated through equations (17) and (18) using the new pair function

$g\left(r, \omega_{1}, \omega_{2}\right)=\exp \left[-\beta u_{\mathrm{HS}}(r)-\beta u\left(r, \omega_{1}, \omega_{2}\right)+\gamma\left(r, \omega_{1}, \omega_{2}\right)+b\left(r, \omega_{1}, \omega_{2}\right)\right]$

and the pair potential $u\left(r_{12}, \omega_{1}, \omega_{2}\right)$.

Expanding $g\left(r_{12}, \omega_{1}, \omega_{2}\right)$ and $u\left(r_{12}, \omega_{1}, \omega_{2}\right)$ in the generalized spherical harmonics and integrating out the $\phi$ dependence, we get for the KBGY relation in $\theta$, equation (17),

$$
\frac{\mathrm{d}}{\mathrm{d} \theta} \ln \left[\frac{f_{z}(\cos \theta)}{f_{0 z}(\cos \theta)}\right]=-\sum_{l_{1}, l_{2}, m} \xi_{l_{1} l_{2}}^{m m} \mathcal{P}_{l_{1} m}(\cos \theta) \frac{\mathrm{d}}{\mathrm{d} \theta} \mathcal{P}_{l_{2} m}(\cos \theta),
$$

where

$$
\xi_{l_{1} l_{2}}^{m_{1} m_{2}} \equiv \rho \int \mathrm{d} r \sum_{l_{3} m_{3}} g_{l_{1} l_{3}}^{m_{1} m_{3}}(r) \beta u_{l_{3} l_{2}}^{m_{3} m_{2}}(r) .
$$

The actual nonzero potential coefficients restrict $\left(l_{2}, m\right)$ in the sum of equation (64) to the set $(0,0),(2,0),(2,1),(2,2)$. This equation can then be integrated to give

$$
\begin{aligned}
& \ln f_{z}(\cos \theta)=\ln f_{0 z}(\cos \theta)-F_{z}(\cos \theta)+\text { constant, } \\
& F_{z}(x)=\xi_{02}^{00} \mathcal{P}_{20}(x)+\frac{1}{2} \xi_{22}^{00} \mathcal{P}_{20}^{2}(x)+\xi_{22}^{11} \mathcal{P}_{21}^{2}(x)+\xi_{22}^{22} \mathcal{P}_{22}^{2}(x)+\cdots
\end{aligned}
$$

Coefficients $\xi_{l_{1} l_{2}}^{m_{1} m_{2}}$ with indices $l_{j} \geqslant 4$ in the ellipsis will be neglected. The new distribution function $f_{z}(\cos \theta)$ is finally found as

$$
\begin{aligned}
& \ln f_{z}(\cos \theta)=\ln f_{0 z}(x)-\sum_{l \geqslant 1} a_{l} \mathcal{P}_{l 0}(\cos \theta)+\text { constant, } \\
& a_{l}=\frac{1}{2} \int_{0}^{\pi} \mathrm{d} \theta \sin \theta f_{z}(\cos \theta) F_{z}(\cos \theta) \mathcal{P}_{l 0}(\cos \theta) .
\end{aligned}
$$

Odd coefficients $a_{1}, a_{3}, \ldots$ vanish. Further, we find that for the thermodynamic states considered in this work the coefficient $a_{4}$ is at least two orders of magnitude smaller than $a_{2}$, so that, keeping only $a_{2}, f_{z}(\cos \theta)$ retains the form of $f_{0 z}(\cos \theta)$,

$$
\ln f_{z}(\cos \theta)=-\frac{3}{2} \beta W \cos ^{2} \theta+\text { constant, }
$$

with an interaction-altered field strength

$$
W=W_{0}+\frac{2 a_{2}}{3 \beta\left(m_{4}-m_{2}^{2}\right)^{1 / 2}} .
$$

The constant in equation (70) is determined by normalization (see equation (10)); integrals (69) are evaluated numerically and exactly using Gaussian quadrature,

$$
a_{l}=\sum_{j=1}^{n} w\left(x_{j}\right) F_{z}\left(x_{j}\right) \mathcal{P}_{l 0}\left(x_{j}\right)
$$


with $l<n$, where the $x_{j}$ are the $n$ zeros of $\mathcal{P}_{n 0}(x)$ and $w\left(x_{j}\right)$ the corresponding Gaussian weights. These quantities, along with the generalized Legendre functions themselves, are calculated using a robust algorithm proposed by Press and Teukolsky [24]. All Gaussian quadratures in the calculation were carried out using $n=10$ Gaussian root points.

Calculation of $f_{x}(\cos \phi)$ from the KBGY relation in $\phi$, equation (18), runs along similar lines. Again expanding the pair functions in generalized spherical harmonics and now integrating out the $\theta$ dependence, we arrive finally at

$$
\begin{aligned}
& \ln f_{x}(\cos \phi)= \ln f_{0 x}(\cos \phi)-F_{x}(\cos \phi)+\text { constant, } \\
& F_{x}(y)=\xi_{02}^{02}\left\langle\mathcal{P}_{22}(x)\right\rangle\left[\mathcal{E}_{2}(y)+\mathcal{E}_{2}^{*}(y)\right]+\xi_{22}^{02}\left\langle\mathcal{P}_{20}(x) \mathcal{P}_{22}(x)\right\rangle\left[\mathcal{E}_{2}(y)+\mathcal{E}_{2}^{*}(y)\right]+\xi_{22}^{11}\left|\mathcal{E}_{1}(y)\right|^{2} \\
& \quad+\frac{1}{2} \xi_{22}^{1-1}\left[\mathcal{E}_{1}^{2}(y)+\mathcal{E}_{1}^{* 2}(y)\right]+\xi_{22}^{22}\left|\mathcal{E}_{2}(y)\right|^{2}+\frac{1}{2} \xi_{22}^{2-2}\left[\mathcal{E}_{2}^{2}(y)+\mathcal{E}_{2}^{* 2}(y)\right]+\cdots,
\end{aligned}
$$

and again coefficients $\xi_{l_{1} l_{2}}^{m_{1} m_{2}}$ with indices $l_{j} \geqslant 4$ in the ellipsis will be neglected. The averaged quantities in equation $(74)$ are $\left\langle\mathcal{P}_{22}(x)\right\rangle=\left(1-m_{2}\right) /\left(1-2 m_{2}+m_{4}\right)^{1 / 2}$ and $\left\langle\mathcal{P}_{20}(x) \mathcal{P}_{22}(x)\right\rangle=-\left[\left(m_{4}-m_{2}^{2}\right) /\left(1-2 m_{2}+m_{4}\right)\right]^{1 / 2}$. The new distribution function $f_{x}(\cos \phi)$ is then found as

$$
\begin{aligned}
& \ln f_{x}(\cos \phi)=\ln f_{0 x}(\cos \phi)-\sum_{m} b_{m} \mathcal{E}_{m}(\cos \phi)+\text { constant, } \\
& b_{m}=\frac{1}{2 \pi} \int_{0}^{2 \pi} \mathrm{d} \phi f_{x}(\cos \phi) F_{x}(\cos \phi) \mathcal{E}_{m}^{*}(\cos \phi) .
\end{aligned}
$$

Odd coefficients $b_{1}, b_{3}, \ldots$, vanish and, again in practice, we find that for the states considered herein the coefficient $b_{2}$ is at least two orders of magnitude larger than $b_{4}$, so that $f_{x}(\cos \phi)$ also effectively retains the form of $f_{0 x}(\cos \phi)$,

$$
\ln f_{x}(\cos \phi)=\beta A \cos 2 \phi+\text { constant, }
$$

but with an interaction-altered field strength

$$
A=A_{0}-\frac{2 b_{2}}{\beta\left(1-2 \mu_{2}^{2}+\mu_{4}\right)^{1 / 2}} .
$$

The constant in equation (77) is determined by normalization (see equation (11)); integrals (76) are evaluated numerically and exactly using 10-point Gaussian quadrature.

Successive rounds of iterations for $\gamma\left(r_{12}, \omega_{1}, \omega_{2}\right)$ and $f(\omega)=f_{z}(\cos \theta) f_{x}(\cos \phi)$ are repeated until both functions are self-consistently converged.

\section{Thermodynamics and structure}

Once the one-body and two-body distribution functions have been determined, it is straightforward to calculate the various thermodynamic quantities. For instance, the excess internal energy $U$ and the pressure $p$ are found as quadratures,

$$
\begin{aligned}
& \frac{\beta U}{N}=\frac{1}{2} \rho \int \mathrm{d} \boldsymbol{r} \sum_{l_{1}, l_{2}, m_{1}, m_{2}} g_{l_{1} l_{2}}^{m_{1} m_{2}}(r) \beta u_{l_{1} l_{2}}^{m_{1} m_{2}}(r), \\
& \frac{\beta p}{\rho}=1+\frac{1}{2} \pi \rho \sigma^{2} g_{00}^{00}(\sigma)-\frac{1}{4} \rho \int \mathrm{d} \boldsymbol{r} \sum_{l_{1}, l_{2}, m_{1}, m_{2}} g_{l_{1} l_{2}}^{m_{1} m_{2}}(r) r \frac{\mathrm{d}}{\mathrm{d} r} \beta u_{l_{1} l_{2}}^{m_{1} m_{2}}(r),
\end{aligned}
$$

in terms of the coefficients of the expansions of the pair distribution function and the potential function in generalized spherical harmonics, while the isothermal compressibility $\chi_{T}$ is given by

$$
\rho k_{\mathrm{B}} T \chi_{T}=1+\rho \tilde{h}_{00}^{00}(0)
$$


where $h=g-1$. The thermodynamic integrals above were evaluated with the trapezoidal rule for unequal intervals at the points defined by the Hankel transform operations.

The response of the system to the external fields is described by the 'polarizations'

$$
\begin{aligned}
& M_{z}=\frac{1}{\beta} \frac{\partial \ln Z}{\partial W_{0}}=-\frac{1}{2} N\left(3 m_{2}-1\right), \\
& M_{x}=\frac{1}{\beta} \frac{\partial \ln Z}{\partial A_{0}}=N \mu_{2} .
\end{aligned}
$$

The susceptibilities are then

$$
\chi_{z z}=\frac{1}{A} \frac{\partial M_{z}}{\partial W_{0}}, \quad \chi_{x x}=\frac{1}{A} \frac{\partial M_{x}}{\partial A_{0}}, \quad \chi_{x z}=\chi_{z x}=\frac{1}{A} \frac{\partial M_{x}}{\partial W_{0}} .
$$

We find for these

$$
\begin{aligned}
\chi_{z z} / \beta \rho=\frac{1}{4}(3 & \left.m_{2}-1\right)^{2}\left[1+\rho \tilde{h}_{00}^{00}(0)\right]+\frac{3}{2}\left(3 m_{2}-1\right)\left(m_{4}-m_{2}^{2}\right)^{1 / 2} \rho \tilde{h}_{20}^{00}(0) \\
+ & \frac{9}{4}\left(m_{4}-m_{2}^{2}\right)\left[1+\rho \tilde{h}_{22}^{00}(0)\right] \\
\chi_{x x} / \beta \rho=\mu_{2}^{2}[1 & \left.+\rho \tilde{h}_{00}^{00}(0)\right]+2 \mu_{2}\left(1-2 \mu_{2}^{2}+\mu_{4}\right)^{1 / 2}\left\langle\mathcal{P}_{22}(x)\right\rangle \rho \tilde{h}_{20}^{20}(0) \\
+ & \frac{1}{2}\left(1-2 \mu_{2}^{2}+\mu_{4}\right)\left\{1+\left\langle\mathcal{P}_{22}(x)\right\rangle^{2}\left[\rho \tilde{h}_{22}^{22}(0)+\rho \tilde{h}_{22}^{2-2}(0)\right]\right\}+\cdots \\
-\chi_{x z} / \beta \rho=\frac{1}{2} & \mu_{2}\left(3 m_{2}-1\right)\left[1+\rho \tilde{h}_{00}^{00}(0)\right]+\frac{3}{2} \mu_{2}\left(m_{4}-m_{2}^{2}\right)^{1 / 2} \rho \tilde{h}_{20}^{00}(0) \\
& +\left(1-2 \mu_{2}^{2}+\mu_{4}\right)^{1 / 2}\left\langle\mathcal{P}_{22}(x)\right\rangle\left[\left(3 m_{2}-1\right) \rho \tilde{h}_{20}^{20}(0)\right. \\
+ & \left.3\left(m_{4}-m_{2}^{2}\right)^{1 / 2} \rho \tilde{h}_{22}^{20}(0)\right]+\cdots
\end{aligned}
$$

where the terms in the ellipses will be neglected.

The structure of the liquid is succinctly described by the angle-averaged distribution functions

$$
\begin{aligned}
G_{l}(r) & =(2 l+1)\left\langle g\left(r, \omega_{1}, \omega_{2}\right) P_{l}\left(\cos \theta_{12}\right)\right\rangle_{\omega_{1} \omega_{2}} \\
& \equiv \frac{2 l+1}{(4 \pi)^{2}} \int \mathrm{d} \omega_{1} \omega_{2} f\left(\omega_{1}\right) f\left(\omega_{2}\right) g\left(r, \omega_{1}, \omega_{2}\right) P_{l}\left(\cos \theta_{12}\right) .
\end{aligned}
$$

Key among these are

$$
G_{0}(r)=g_{00}^{00}(r)
$$

which is a measure of the relative density of particles at centre-to-centre distance $r$ about a central particle, and

$$
G_{2}(r)=5 \sum_{l_{1}, l_{2}, m_{1}, m_{2}} g_{l_{1} l_{2}}^{m_{1} m_{2}}(r) P_{l_{1} l_{2}}^{m_{1} m_{2}},
$$

which is a measure of the average relative orientation between two fluid particles at centreto-centre distance $r$. The external fields induce long-range orientational order, so that for large $r$,

$$
G_{2}(r) \sim 5 P_{00}^{00}=\frac{5}{4}\left(3 m_{2}-1\right)^{2}+\frac{15}{4} \mu_{2}^{2}\left(1-m_{2}\right)^{2} .
$$

In the limit of zero fields $\left(W_{0}=A_{0}=0\right), G_{2}(r)$ becomes the usual rotational-invariant coefficient $g^{220}(r)$,

$$
\left.G_{2}(r)\right\rfloor_{W_{0}=A_{0}=0}=g^{220}(r)=g_{22}^{00}(r)-2 g_{22}^{11}(r)+2 g_{22}^{22}(r),
$$

and goes asymptotically to zero. 


\section{Monte Carlo simulation and order parameters}

The simulation method to be applied depends on the phase behaviour that is expected in each of the cases. We consider systems with $W_{0}=A_{0}=0$ and $W_{0} \neq 0, A_{0}=0$ along with systems in which both external fields are nonzero. The first two regimes, with $A_{0}=0$, will exhibit an orientational order-disorder phase transition, while in the third the presence of both fields removes the transition by inducing orientational order at all temperatures. (Obviously, a condensation transition might still be possible depending on the range of the interaction, but that is outside the scope of this study.) We have then two distinct situations, one in which we have to deal with criticality and another in which we expect the system to behave like an ordinary homogeneous and isotropic bulk fluid. In the latter case (i.e., when both fields are nonzero) we use the usual Monte Carlo algorithm without further elaboration. But in the cases where $A_{0}=0$ one can encounter critical slowing down at low temperatures and therefore, in order to improve the orientational sampling, here we apply a combination of cluster and singleparticle moves. The cluster algorithm used was developed following the ideas of Swendsen and Wang [25] and can be found in full detail in [26] applied to an off-lattice Heisenberg system. For the single-particle moves we have devised an algorithm which guarantees that each and every particle move is accepted [27].

The course of a Monte Carlo simulation can then be sketched as follows. The set of particle moves is organized in sweeps. Each Monte Carlo sweep for a system of $N$ particles consists of $N$ translational moves performed according to a standard Monte Carlo algorithm, followed by either $N$ orientational moves or one cluster move-the type of move alternating with successive sweeps. For the systems studied here, we have carried out a total of $10^{4}$ sweeps during equilibration and $10^{5}$ sweeps in the production run in samples with 900 particles. As mentioned earlier, when both fields are nonzero we use a standard Monte Carlo algorithm which combines rotational and translational moves, again generating $10^{4}$ configurations during equilibration and $10^{5}$ configurations to calculate the ensemble averages.

Finally, a few words regarding the calculation of the order parameters. In the integral equation formalism we can directly evaluate the moments $m_{2}$ and $\mu_{2}$, which immediately yield the usual nematic order parameters $\left\langle P_{2}(\cos \theta)\right\rangle=\left(3 m_{2}-1\right) / 2$ and $\left\langle T_{2}(\cos \phi)\right\rangle=\mu_{2}$. The coordinate frame for the angles is defined by $\boldsymbol{W}_{0}$ ( $z$ axis) and $\boldsymbol{A}_{0}$ ( $x$ axis). When one or both fields are turned off the equations will 'remember' their direction if the iterations are started from an ordered solution obtained in nonzero field.

Similarly, in a simulation when both $W_{0}$ and $A_{0}$ are nonzero we can directly calculate the MC averages

$$
\begin{aligned}
& \left\langle P_{2}(\cos \theta)\right\rangle=\frac{1}{2 N} \sum_{i=1}^{N}\left\langle 3 s_{i}^{z} s_{i}^{z}-1\right\rangle_{\mathrm{MC}}, \\
& \left\langle T_{2}(\cos \phi)\right\rangle=\frac{1}{N} \sum_{i=1}^{N}\left\langle 2 u_{i}^{x} u_{i}^{x}-1\right\rangle_{\mathrm{MC}}
\end{aligned}
$$

where $\hat{\boldsymbol{u}} \equiv\left(s^{x}, s^{y}\right) /\left[\left(s^{x}\right)^{2}+\left(s^{y}\right)^{2}\right]^{1 / 2}$ is a unit vector in the system plane. When $A_{0}=0$ however there is no longer a special direction in the system plane and equation (94) will average to zero regardless of system ordering. Here we use instead a two-dimensional version of Saupe's tensor [28],

$$
Q_{\alpha \beta}^{2 \mathrm{D}}=\frac{1}{N} \sum_{i=1}^{N}\left\langle 2 u_{i}^{\alpha} u_{i}^{\beta}-\delta_{\alpha \beta}\right\rangle_{\mathrm{MC}}, \quad \text { with } \alpha, \beta=x, y,
$$

whose largest eigenvalue gives the order parameter $S^{2 \mathrm{D}}$. It is easy to recognize that $S^{2 \mathrm{D}}=\mu_{2}$. 
Finally, when $W_{0}=0$ then equation (93) will average to zero, again regardless of system ordering, even though in this case the $z$ axis continues to be uniquely defined as the normal to the system plane. But because the Maier-Saupe potential we are using decouples the axial orientations $\omega$ from the in-plane vectors $r$, the orientations $\omega$ now see no special direction in space. An orientational quantity that can still be calculated by simulation in these circumstances is the Kerr constant, $G_{\mathrm{K}}=\left\langle P_{2}\left(\cos \theta_{12}\right)\right\rangle$, where $\cos \theta_{12}=\hat{s}_{1} \cdot \hat{\boldsymbol{s}}_{2}$. We have then that

$$
\begin{aligned}
G_{\mathrm{K}} & \equiv P_{00}^{00}=\frac{1}{4}\left(3 m_{2}-1\right)^{2}+\frac{3}{4} \mu_{2}^{2}\left(1-m_{2}\right)^{2} \\
& =\left\langle P_{2}(\cos \theta)\right\rangle^{2}+\frac{1}{3} \mu_{2}^{2}\left(1-\left\langle P_{2}(\cos \theta)\right\rangle\right)^{2},
\end{aligned}
$$

which can be inverted to give

$$
\left\langle P_{2}(\cos \theta)\right\rangle=-\frac{\left[9 G_{\mathrm{K}}+3 \mu_{2}^{2}\left(G_{\mathrm{K}}-1\right)\right]^{1 / 2}-\mu_{2}^{2}}{3+\mu_{2}^{2}},
$$

where both $G_{\mathrm{K}}$ and $\mu_{2}$ are known from simulation. When $W_{0} \neq 0$, equations (98) and (93) are equivalent; calculating both serves as an internal check. Similarly, when $A_{0} \neq 0$ the method using equation (95) is equivalent to equation (94) and both can be calculated to check internal consistency.

We note in passing that $G_{\mathrm{K}}$ is also connected with the largest eigenvalue of the threedimensional Saupe tensor [28],

$$
Q_{\alpha \beta}^{3 \mathrm{D}}=\frac{1}{2 N} \sum_{i=1}^{N}\left\langle 3 u_{i}^{\alpha} u_{i}^{\beta}-\delta_{\alpha \beta}\right\rangle_{\mathrm{MC}}, \quad \text { with } \alpha, \beta=x, y, z,
$$

known in simulation as the order parameter $S$, by the simple relation $G_{\mathrm{K}}=S^{2}$. With this and equation (91), one recovers the relation [29]

$$
\lim _{r \rightarrow \infty} G_{2}(r)=5 G_{\mathrm{K}}=5 S^{2},
$$

which is known to be fulfilled in phases with long-range nematic order.

\section{Results}

We report here sample results obtained from the integral equations using two different closures, RHNC and MSA, and from Monte Carlo (MC) simulations for the planar system at reduced density $\rho \sigma^{2}=0.8$, reduced range parameter $\kappa \sigma=1.0$, and a spread of reduced temperatures $T^{*}$.

In the RHNC closure, the bridge function $b\left(r, \omega_{1}, \omega_{2}\right)$ needed in equation (20) is approximated by the known bridge function of some selected reference system, generally a hard core model. For the two-dimensional fluid treated here, we take as reference system a hard disc (HD) fluid at the same density, and put

$$
b\left(r, \omega_{1}, \omega_{2}\right) \approx b_{\mathrm{HD}}(r)
$$

There is no parameterized solution known for the hard disc fluid that maintains internal thermodynamic consistency, and so to get $b_{\mathrm{HD}}(r)$ we resort to an approximate closure for this model that achieves at least pressure consistency [22], namely

$c_{\mathrm{HD}}(r)=h_{\mathrm{HD}}(r)-(1-\mu)\left[g_{\mathrm{HD}}(r) \mathrm{e}^{\beta u_{\mathrm{HD}}(r)}-1\right]-\mu \ln \left[g_{\mathrm{HD}}(r) \mathrm{e}^{\beta u_{\mathrm{HD}}(r)}\right]$.

This is a blend of the familiar Percus-Yevick $(\mu=0)$ and hypernetted chain $(\mu=1)$ closures controlled by the parameter $\mu$ to achieve consistency of the virial and compressibility pressures. The density dependence of $\mu$ constrained in this way is found by calculation to be fitted by

$$
\mu=0.0920+0.1222\left(\rho \sigma^{2}\right)+0.1642\left(\rho \sigma^{2}\right)^{2}+0.1100\left(\rho \sigma^{2}\right)^{3} .
$$




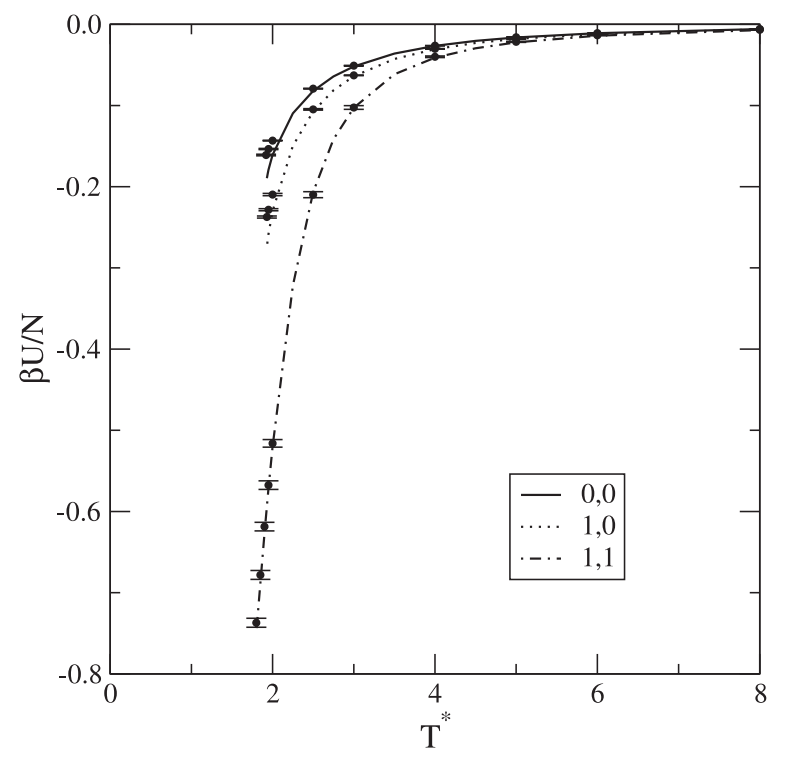

Figure 1. Excess internal energy from the RHNC integral equation (lines) and MC simulation (points) for a planar nematic liquid at $\rho \sigma^{2}=0.8$ with $\kappa \sigma=1.0$ and three external field combinations $W_{0}, A_{0}$ as shown in the legend box.

A better approximation for $b\left(r, \omega_{1}, \omega_{2}\right)$ would include anisotropy with at least a $P_{2}\left(\cos \theta_{12}\right)$ term, as in the pair potential, but essentially nothing is known about such generalized bridge functions.

The MSA is concisely expressed by a pair of equations,

$$
\begin{aligned}
& g\left(r, \omega_{1}, \omega_{2}\right)=0, \quad r<\sigma, \\
& c\left(r, \omega_{1}, \omega_{2}\right)=-\beta u\left(r, \omega_{1}, \omega_{2}\right), \quad r>\sigma .
\end{aligned}
$$

The first of these is of course exact; the approximation is in the second equation, which, by linearizing equation (20), enormously reduces the computing time for the iteration cycle in (50) compared to RHNC.

Table 1 summarizes the principal thermodynamic data obtained with the RHNC closure. Q.1 The effective fields $W$ and $A$ define the calculated one-body distribution functions and show how the tendency of the pair interaction to mutually align molecular axes can multiply the effect of the external fields on an individual molecule. With decreasing temperature the molecular axes become more aligned with each other and the system becomes more energetically bound. This is seen graphically in figure 1, with the excess energy $U$ dropping more and more steeply as first the disorienting field $\boldsymbol{W}_{0}$ and then the in-plane orienting field $\boldsymbol{A}_{0}$ are turned on. The drop in pressure with temperature in table 1 is steady but not as dramatic as that of $U$. Comparison of $p$ and $U$ with MC values in table 1 shows that the present isotropic implementation of RHNC performs reasonably well but falls short of what is usually attained with this closure in simple liquids; evidently the orientational part of $b\left(r, \omega_{1}, \omega_{2}\right)$ is not negligible.

The increase in orientational order with decreasing temperature in the presence of both fields is seen in figure 2; one can readily visualize these lines as $T^{*} \rightarrow 0$ extending toward perfect order, which is $-1 / 2$ for $\left\langle P_{2}(\cos \theta)\right\rangle$ and +1 for $\left\langle T_{2}(\cos \phi)\right\rangle$. Figure 3 displays pair structure in the planar liquid. The function $G_{0}(r)$ is just the angle-averaged $g\left(r, \omega_{1}, \omega_{2}\right)$ and changes little across the states in table 1 for the fixed density $\rho \sigma^{2}=0.8$; we show the specific 
Table 1. Results computed using the RHNC integral equation and MC simulation (900 particles) for a planar nematic liquid at $\rho \sigma^{2}=0.8$ with $\kappa \sigma=1.0$ and three external field combinations $W_{0}, A_{0}$. For $A_{0}=0$ states, converged RHNC solutions cannot be obtained appreciably below the lowest listed temperature. 
Table 1. (Continued.)

\begin{tabular}{|c|c|c|c|c|c|c|c|c|c|c|c|c|c|c|c|}
\hline \multirow[b]{2}{*}{$T^{*}$} & \multirow[b]{2}{*}{$W_{0} / K$} & \multirow[b]{2}{*}{$W / K$} & \multirow[b]{2}{*}{$A_{0} / K$} & \multirow[b]{2}{*}{$A / K$} & \multicolumn{2}{|c|}{$\beta p / \rho$} & \multicolumn{2}{|c|}{$\beta U / N$} & \multicolumn{2}{|c|}{$\left\langle P_{2}(\cos \theta)\right\rangle$} & \multicolumn{2}{|c|}{$\left\langle T_{2}(\cos \phi)\right\rangle$} & \multirow[b]{2}{*}{$v_{1}(0)$} & \multirow[b]{2}{*}{$v_{2}(0)$} & \multirow[b]{2}{*}{$\nu_{3}(0)$} \\
\hline & & & & & RHNC & $\mathrm{MC}$ & RHNC & $\mathrm{MC}$ & RHNC & $\mathrm{MC}$ & RHNC & MC & & & \\
\hline 8.00 & 1 & 1.209 & 1 & 1.172 & 7.263 & $7.440(34)$ & -0.0071 & $-0.0068(04)$ & -0.0296 & -0.0303 & 0.0731 & 0.0756 & 1.180 & 1.213 & 1.211 \\
\hline 6.00 & 1 & 1.305 & 1 & 1.250 & 7.257 & $7.432(29)$ & -0.0140 & $-0.0136(05)$ & -0.0421 & -0.0422 & 0.1036 & 0.1090 & 1.243 & 1.313 & 1.308 \\
\hline 5.00 & 1 & 1.395 & 1 & 1.322 & 7.247 & $7.422(34)$ & -0.0223 & $-0.0216(07)$ & -0.0535 & -0.0538 & 0.1311 & 0.1386 & 1.292 & 1.409 & 1.400 \\
\hline 4.00 & 1 & 1.562 & 1 & 1.454 & 7.226 & $7.406(42)$ & -0.0411 & $-0.0400(10)$ & -0.0735 & -0.0731 & 0.1789 & 0.1908 & 1.361 & 1.589 & 1.571 \\
\hline 3.00 & 1 & 1.966 & 1 & 1.770 & 7.152 & $7.328(29)$ & -0.1028 & $-0.1025(23)$ & -0.1177 & -0.1176 & 0.2829 & 0.3065 & 1.442 & 2.048 & 1.994 \\
\hline 2.50 & 1 & 2.471 & 1 & 2.159 & 7.022 & $7.207(23)$ & -0.2067 & $-0.2099(37)$ & -0.1666 & -0.1661 & 0.3960 & 0.4282 & 1.451 & 2.660 & 2.535 \\
\hline 2.00 & 1 & 3.522 & 1 & 2.967 & 6.617 & $6.829(27)$ & -0.5201 & $-0.5161(47)$ & -0.2540 & -0.2504 & 0.5920 & 0.6118 & 1.390 & 4.072 & 3.686 \\
\hline 1.95 & 1 & 3.655 & 1 & 3.068 & 6.549 & $6.769(36)$ & -0.5715 & $-0.5675(53)$ & -0.2641 & -0.2609 & 0.6138 & 0.6319 & 1.383 & 4.271 & 3.835 \\
\hline 1.90 & 1 & 3.788 & 1 & 3.172 & 6.478 & $6.704(37)$ & -0.6269 & $-0.6186(53)$ & -0.2742 & -0.2706 & 0.6353 & 0.6499 & 1.378 & 4.480 & 3.986 \\
\hline 1.85 & 1 & 3.921 & 1 & 3.275 & 6.400 & $6.619(25)$ & -0.6862 & $-0.6781(55)$ & -0.2841 & -0.2808 & 0.6562 & 0.6707 & 1.375 & 4.699 & 4.137 \\
\hline 1.80 & 1 & 4.051 & 1 & 3.375 & 6.317 & $6.549(35)$ & -0.7489 & $-0.7370(55)$ & -0.2937 & -0.2901 & 0.6761 & 0.6875 & 1.373 & 4.934 & 4.291 \\
\hline
\end{tabular}




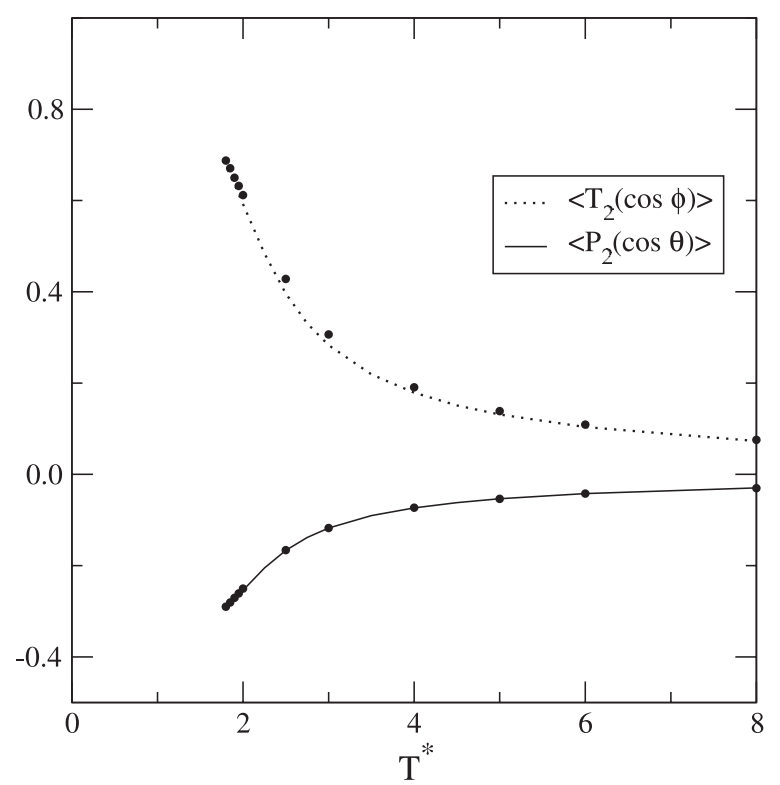

Figure 2. Order parameters $\left\langle P_{2}(\cos \theta)\right\rangle$ and $\left\langle T_{2}(\cos \phi)\right\rangle$ from the RHNC integral equation (lines) and $\mathrm{MC}$ simulation (points) for a planar nematic liquid at $\rho \sigma^{2}=0.8$ with $\kappa \sigma=1.0$ and external fields $W_{0}=A_{0}=1.0$.

values at an intermediate temperature, $T^{*}=3$. The tendency of near neighbours to align with each other is seen in $G_{2}(r)$ to be much more sensitive to changes in temperature and external field.

If any of the eigenvalues $\lambda_{j}(k)$, equations (53)-(55), should vanish then the matrix OZ equation would have no inverse and the integral equation no solution. Following the stability analysis of Chen et al $[30,31]$, this signals a phase change. We see in figure 4 that $\lambda_{3}(0)$ indeed tends towards zero with decreasing temperature if $A_{0}=0$, more rapidly in the presence of a disorienting field $W_{0}$. This is consistent with the expectation discussed earlier that the isotropic-nematic transition occurs at a higher temperature in the presence of a disorienting field. Note that in zero field $\lambda_{1}(0)=\lambda_{2}(0)=\lambda_{3}(0)$, while with $W_{0} \neq 0, A_{0}=0$ we have $\lambda_{1}(0)=\lambda_{2}(0) \neq \lambda_{3}(0)$. Only with both fields turned on are the three eigenvalues nondegenerate and in figure 4 the change of slope in $\lambda_{3}(0)$ in the presence of both fields at low temperatures indicates that in this case the phase transition has vanished. Figure 5 displays the eigenvalue inverses $v_{j}(k)=1 / \lambda_{j}(k)$, equations (58)-(60); they are relatively featureless, with significant values only for small $k$. (Values at $k=0$ are in table 1.) The curves shown are for $T^{*}=2.0$ with $W_{0}=1$ and $A_{0}=0$.

The two order parameters show that the states in table 1 with $A_{0}=1$ are highly ordered at low temperatures. One might hope that using such a low-temperature solution as input for iterations with $A_{0}=0$ would allow the system to relax into an ordered state that maintained $A>0$. This does not work with the RHNC closure; the iterations do not converge and no solution is found.

We turn then to the MSA. Table 2 summarizes the principal thermodynamic data obtained with the MSA closure. Qualitatively the results at higher temperatures are similar to those of RHNC. Quantitatively the pressure and energy are notably poorer while the order parameters are remarkably just as good. The principal differences however are seen at lower temperatures. 


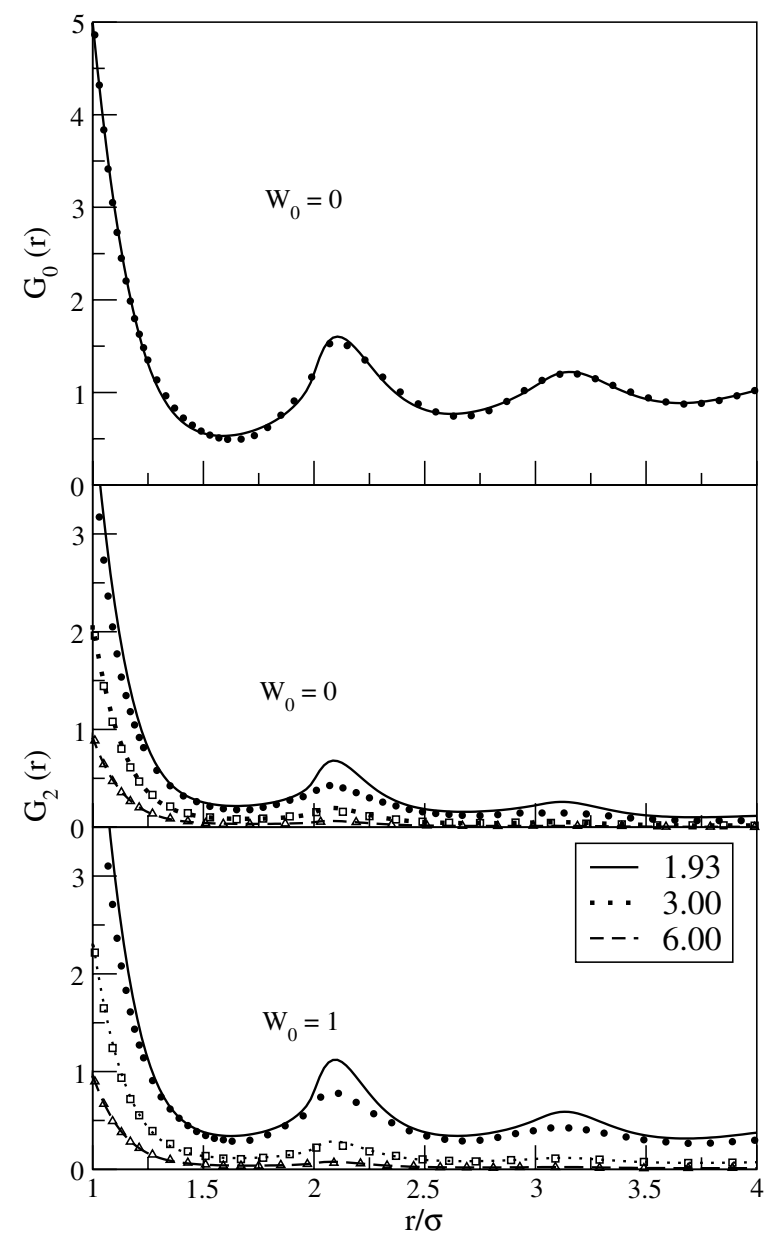

Figure 3. Centre-to-centre distribution function $G_{0}(r)$ in zero field and angular projection $G_{2}(r)$ without and with a disorienting field from the RHNC integral equation (lines) and MC simulation (symbols) for a planar nematic liquid at $\rho \sigma^{2}=0.8$ with $\kappa \sigma=1.0$. Values in the legend box indicate the temperatures $T^{*}$ at which the calculations were performed.

Here we are able to carry out the program suggested above: start iterations for $A_{0}=0$ using the solutions found for $A_{0}=1$ and allow the system to find its equilibrium value of the effective $A$ field. (Starting from a solution with any $A_{0}>0$ will work the same. Further, only the lowest temperature state need be solved in this way; higher temperature cases can then start from a lower temperature solution with $A_{0}=0$.) We see that indeed the system remains in an ordered state, with the values of $A$ and $\left\langle T_{2}(\cos \phi)\right\rangle$ dropping as temperature rises until a critical temperature is reached and the system shifts into a disordered state. This happens with and without a disorienting field $W_{0}$, but the transition temperature is clearly higher with the disorienting field.

One important point must be stressed here regarding the order parameters. The reported simulation values of $\left\langle T_{2}(\cos \phi)\right\rangle$ when $A_{0}=0$ and $\left\langle T_{2}(\cos \phi)\right\rangle$ and $\left\langle P_{2}(\cos \theta)\right\rangle$ when $W_{0}=A_{0}=0$ are representative only of the 900-particle samples. As we will show in a later paper [27], there is a BKT [19, 20] defect-mediated continuous transition without true 
Table 2. Results computed using the MSA integral equation and MC simulation ( 900 particles) for a planar nematic liquid at $\rho \sigma^{2}=0.8$ with $\kappa \sigma=1.0$ and three external field combinations $W_{0}, A_{0}$. Note that the simulation values of $\left\langle P_{2}(\cos \theta)\right\rangle$ and $\left\langle T_{2}(\cos \phi)\right\rangle$ when $A_{0}=W_{0}=0$ and those of $\left\langle T_{2}(\cos \phi)\right\rangle$ when just $A_{0}=0$ are representative only of the 900-particle sample, since these quantities should vanish in the thermodynamic limit for the quasiordered states of the BKT phase.

\begin{tabular}{|c|c|c|c|c|c|c|c|c|c|c|c|c|c|c|c|}
\hline \multirow[b]{2}{*}{$T^{*}$} & \multirow[b]{2}{*}{$W_{0} / K$} & \multirow[b]{2}{*}{$W / K$} & \multirow[b]{2}{*}{$A_{0} / K$} & \multirow[b]{2}{*}{$A / K$} & \multicolumn{2}{|c|}{$\beta p / \rho$} & \multicolumn{2}{|c|}{$\beta U / N$} & \multicolumn{2}{|c|}{$\left\langle P_{2}(\cos \theta)\right\rangle$} & \multicolumn{2}{|c|}{$\left\langle T_{2}(\cos \phi)\right\rangle$} & \multirow[b]{2}{*}{$v_{1}(0)$} & \multirow[b]{2}{*}{$v_{2}(0)$} & \multirow[b]{2}{*}{$v_{3}(0)$} \\
\hline & & & & & MSA & MC & MSA & $\mathrm{MC}$ & MSA & MC & MSA & $\mathrm{MC}$ & & & \\
\hline 8.00 & 0 & 0 & 0 & 0 & 6.824 & $7.439(28)$ & -0.0030 & $-0.0059(01)$ & 0 & 0 & 0 & 0 & 1.141 & 1.141 & 1.141 \\
\hline 6.00 & 0 & 0 & 0 & 0 & 6.822 & 7.448(18) & -0.0054 & $-0.0109(02)$ & 0 & 0 & 0 & 0 & 1.196 & 1.196 & 1.196 \\
\hline 5.00 & 0 & 0 & 0 & 0 & 6.819 & 7.431(29) & -0.0078 & $-0.0160(02)$ & 0 & 0 & 0 & 0 & 1.244 & 1.244 & 1.244 \\
\hline 4.00 & 0 & 0 & 0 & 0 & 6.813 & $7.428(25)$ & -0.0125 & $-0.0263(02)$ & 0 & 0 & 0 & 0 & 1.322 & 1.322 & 1.322 \\
\hline 3.00 & 0 & 0 & 0 & 0 & 6.800 & $7.400(37)$ & -0.0230 & $-0.0510(04)$ & 0 & 0 & 0 & 0 & 1.472 & 1.472 & 1.472 \\
\hline 2.00 & 0 & 0 & 0 & 0 & 6.759 & $7.322(31)$ & -0.0559 & $-0.1433(05)$ & 0 & 0 & 0 & 0 & 1.881 & 1.881 & 1.881 \\
\hline 1.50 & 0 & 0 & 0 & 0 & 6.694 & $7.072(25)$ & -0.1079 & $-0.3721(15)$ & 0 & -0.080 & 0 & 0.083 & 2.525 & 2.525 & 2.525 \\
\hline 1.40 & 0 & 2.519 & 0 & 1.900 & 5.966 & $6.907(27)$ & -0.6471 & $-0.5186(39)$ & -0.257 & -0.123 & 0.559 & 0.122 & 1.282 & 3.330 & 3.512 \\
\hline 1.20 & 0 & 3.953 & 0 & 3.001 & 4.936 & $5.892(24)$ & -1.4116 & $-1.3215(29)$ & -0.351 & -0.404 & 0.765 & 0.497 & 1.116 & 3.230 & 3.303 \\
\hline 1.00 & 0 & 4.603 & 0 & 3.479 & 3.997 & $4.944(21)$ & -2.1079 & $-2.0790(18)$ & -0.392 & -0.468 & 0.840 & 0.635 & 1.073 & 3.226 & 3.269 \\
\hline 8.00 & 1 & 1.195 & 0 & 0 & 6.824 & $7.439(26)$ & -0.0033 & $-0.0062(02)$ & -0.0292 & -0.0293 & 0 & 0 & 1.138 & 1.138 & 1.148 \\
\hline 6.00 & 1 & 1.275 & 0 & 0 & 6.820 & 7.435(30) & -0.0063 & $-0.0117(02)$ & -0.0412 & -0.0409 & 0 & 0 & 1.189 & 1.189 & 1.210 \\
\hline 5.00 & 1 & 1.345 & 0 & 0 & 6.816 & 7.437(33) & -0.0096 & $-0.0178(03)$ & -0.0516 & -0.0516 & 0 & 0 & 1.232 & 1.232 & 1.267 \\
\hline 4.00 & 1 & 1.461 & 0 & 0 & 6.807 & 7.413(27) & -0.0165 & $-0.0302(03)$ & -0.0690 & -0.0690 & 0 & 0 & 1.300 & 1.300 & 1.364 \\
\hline 3.00 & 1 & 1.689 & 0 & 0 & 6.784 & 7.381(29) & -0.0348 & $-0.0629(04)$ & -0.1027 & -0.1032 & 0 & 0 & 1.417 & 1.417 & 1.578 \\
\hline 2.00 & 1 & 2.256 & 0 & 0 & 6.679 & $7.232(31)$ & -0.1149 & $-0.2097(14)$ & -0.1850 & -0.1894 & 0 & 0.063 & 1.639 & 1.639 & 2.390 \\
\hline 1.70 & 1 & 2.583 & 0 & 0 & 6.579 & $7.059(33)$ & -0.1912 & $-0.3827(15)$ & -0.2303 & -0.2431 & 0 & 0.105 & 1.728 & 1.728 & 3.303 \\
\hline 1.60 & 1 & 3.351 & 0 & 1.415 & 6.249 & $6.886(28)$ & -0.4360 & $-0.5001(21)$ & -0.2822 & -0.2675 & 0.404 & 0.159 & 1.324 & 2.193 & 3.509 \\
\hline 1.40 & 1 & 4.687 & 0 & 2.690 & 5.427 & $6.278(22)$ & -1.0452 & $-0.9953(29)$ & -0.3531 & -0.3308 & 0.684 & 0.543 & 1.134 & 2.375 & 3.306 \\
\hline 1.20 & 1 & 5.379 & 0 & 3.250 & 4.696 & $5.568(30)$ & -1.5873 & $-1.5686(24)$ & -0.3889 & -0.3756 & 0.786 & 0.715 & 1.084 & 2.424 & 3.258 \\
\hline 1.00 & 1 & 5.866 & 0 & 3.619 & 3.825 & $4.738(32)$ & -2.2344 & $-2.2341(15)$ & -0.4148 & -0.4064 & 0.847 & 0.802 & 1.057 & 2.460 & 3.253 \\
\hline
\end{tabular}


Table 2. (Continued.)

\begin{tabular}{|c|c|c|c|c|c|c|c|c|c|c|c|c|c|c|c|}
\hline \multirow[b]{2}{*}{$T^{*}$} & \multirow[b]{2}{*}{$W_{0} / K$} & \multirow[b]{2}{*}{$W / K$} & \multirow[b]{2}{*}{$A_{0} / K$} & \multirow[b]{2}{*}{$A / K$} & \multicolumn{2}{|c|}{$\beta p / \rho$} & \multicolumn{2}{|c|}{$\beta U / N$} & \multicolumn{2}{|c|}{$\left\langle P_{2}(\cos \theta)\right\rangle$} & \multicolumn{2}{|c|}{$\left\langle T_{2}(\cos \phi)\right\rangle$} & \multirow[b]{2}{*}{$v_{1}(0)$} & \multirow[b]{2}{*}{$v_{2}(0)$} & \multirow[b]{2}{*}{$\nu_{3}(0)$} \\
\hline & & & & & MSA & MC & MSA & MC & MSA & MC & MSA & MC & & & \\
\hline 8.00 & 1 & 1.209 & 1 & 1.172 & 6.823 & $7.440(34)$ & -0.0041 & $-0.0068(04)$ & -0.0296 & -0.0303 & 0.0731 & 0.0756 & 1.127 & 1.149 & 1.148 \\
\hline 6.00 & 1 & 1.306 & 1 & 1.250 & 6.817 & $7.432(29)$ & -0.0085 & $-0.0136(05)$ & -0.0421 & -0.0422 & 0.1036 & 0.1090 & 1.167 & 1.212 & 1.209 \\
\hline 5.00 & 1 & 1.397 & 1 & 1.323 & 6.811 & $7.422(34)$ & -0.0140 & $-0.0216(07)$ & -0.0535 & -0.0538 & 0.1312 & 0.1386 & 1.196 & 1.269 & 1.265 \\
\hline 4.00 & 1 & 1.566 & 1 & 1.458 & 6.794 & $7.406(42)$ & -0.0272 & $-0.0400(10)$ & -0.0736 & -0.0731 & 0.1793 & 0.1908 & 1.234 & 1.368 & 1.360 \\
\hline 3.00 & 1 & 1.988 & 1 & 1.788 & 6.729 & $7.328(29)$ & -0.0759 & $-0.1025(23)$ & -0.1188 & -0.1176 & 0.2856 & 0.3065 & 1.264 & 1.580 & 1.559 \\
\hline 2.00 & 1 & 3.769 & 1 & 3.142 & 6.171 & $6.829(27)$ & -0.4912 & $-0.5161(47)$ & -0.2650 & -0.2504 & 0.6132 & 0.6118 & 1.155 & 2.103 & 2.037 \\
\hline 1.80 & 1 & 4.371 & 1 & 3.590 & 5.856 & $6.549(35)$ & -0.7257 & $-0.7370(55)$ & -0.3058 & -0.2901 & 0.6968 & 0.6875 & 1.118 & 2.203 & 2.123 \\
\hline 1.60 & 1 & 4.917 & 1 & 3.991 & 5.466 & $6.208(28)$ & -1.0152 & $-1.0206(38)$ & -0.3412 & -0.3274 & 0.7644 & 0.7530 & 1.089 & 2.279 & 2.185 \\
\hline 1.40 & 1 & 5.381 & 1 & 4.330 & 4.992 & $5.756(25)$ & -1.3668 & $-1.3744(37)$ & -0.3710 & -0.3600 & 0.8166 & 0.8064 & 1.067 & 2.238 & 2.232 \\
\hline 1.20 & 1 & 5.772 & 1 & 4.614 & 4.088 & $5.204(25)$ & -1.8092 & $-1.8201(25)$ & -0.3963 & -0.3877 & 0.8573 & 0.8484 & 1.052 & 2.388 & 2.271 \\
\hline 1.00 & 1 & 6.105 & 1 & 4.855 & 3.598 & $4.476(21)$ & -2.4019 & $-2.4180(25)$ & -0.4181 & -0.4116 & 0.8899 & 0.8830 & 1.039 & 2.432 & 2.305 \\
\hline
\end{tabular}




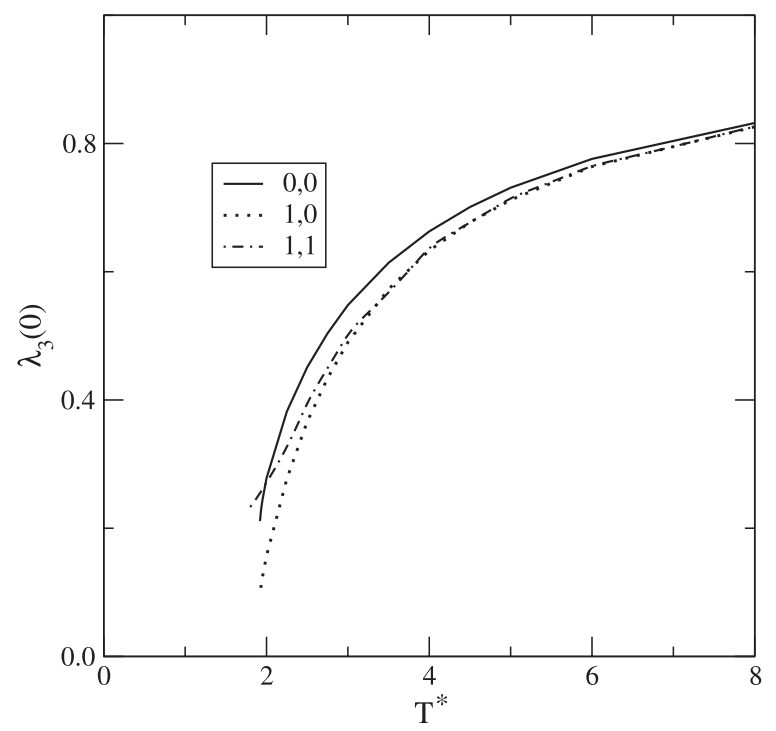

Figure 4. Eigenvalue $\lambda_{3}(k=0)$ from the RHNC integral equation for a planar nematic liquid at $\rho \sigma^{2}=0.8$ with $\kappa \sigma=1.0$ and three external field combinations $W_{0}, A_{0}$ as shown in the legend box.

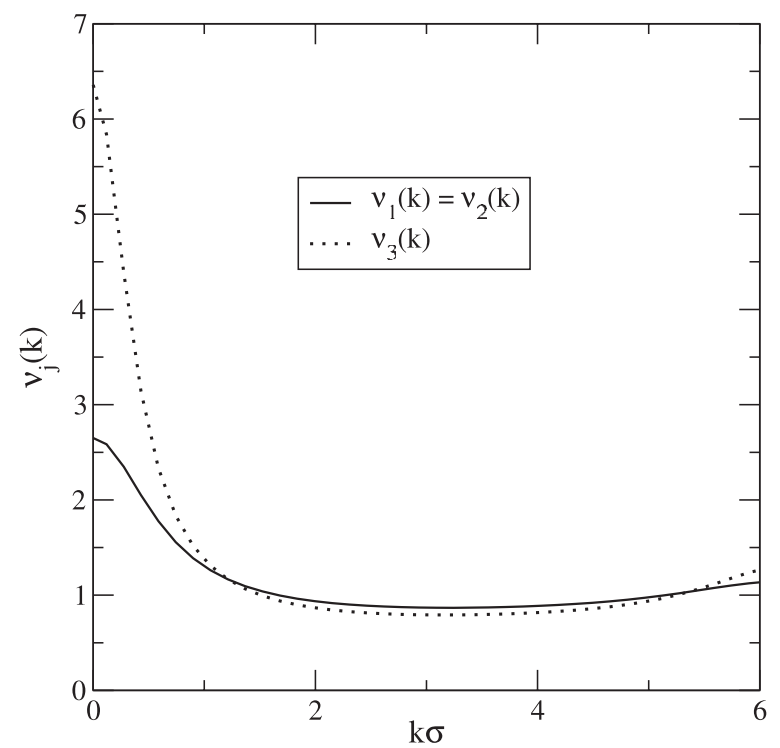

Figure 5. Eigenvalue inverses $v_{1}(k)=v_{2}(k)$ and $v_{3}(k)$ from the RHNC integral equation for a planar nematic liquid at $\rho \sigma^{2}=0.8$ and $T^{*}=2.0$ with $\kappa \sigma=1.0$ and external fields $W_{0}=1$, $A_{0}=0$.

long-range order (although below the transition temperature the system exhibits a large degree of local ordering) by which in the thermodynamic limit the order parameters should vanish. The fact that we encounter values substantially different from zero is a finite-size effect, and it allows us to monitor the onset of the transition by signalling the build-up of local order. The size dependence of structural properties is mostly reflected in the long-range behaviour of 
$G_{2}(r)$, controlled by the order parameters through equation (100), which becomes apparent for distances beyond $10 \sigma$. This in turn implies that the size dependence of other thermodynamic quantities such as the internal energy or the pressure is relatively small and the values of these quantities reported in table 2 are representative of the infinite system.

Obviously, the comparison with the low-temperature MSA results is conditioned by the previous discussion. The MSA approximation is known to predict for this system a weak first-order phase transition [32], by which at sufficiently low temperatures we encounter truly ordered states with nonvanishing order parameters, as seen in table 2. Thus, a comparison of MSA with MC results can only be made for those thermodynamic properties with little size dependence (energy and pressure) and for the approximate location of the transition. As mentioned earlier, and in agreement with the RHNC prediction, the presence of the disorienting field raises the MSA transition temperature. The internal energies below the transition temperature are reproduced with reasonable accuracy while the pressure is somewhat poor, as should be expected for the MSA. It is also worth mentioning that those order parameters that reflect the field-induced orientational ordering (i.e., true long-range order) are accurately reproduced by the theory, which becomes quite apparent when both fields are nonzero.

\section{Acknowledgments}

The financial support of the Dirección General de Investigación Científica under Grant FIS2004-02954-C03-01 is acknowledged by EL, CM and NGA.

\section{Appendix. Generalized Chebyshev functions}

The prerequisite for construction of the modified Chebyshev functions is the set of quasimoments

$$
\mu_{k} \equiv \frac{1}{\pi} \int_{0}^{\pi} \mathrm{d} \phi f_{x}(\cos \phi) \cos k \phi .
$$

For the distribution

$$
f_{x}(\cos \phi)=\frac{\mathrm{e}^{\beta A \cos 2 \phi}}{I_{0}(\beta A)},
$$

these are obtained as

$$
\mu_{k}= \begin{cases}I_{k / 2}(\beta A) / I_{0}(\beta A), & k \text { even, } \\ 0, & k \text { odd }\end{cases}
$$

In these expressions, $I_{n}(x)$ is the modified Bessel function of order $n$.

Then, in a straightforward application of the Gram-Schmidt method [21], we find for the first four members (all that are needed in this calculation)

$$
\begin{aligned}
\mathcal{T}_{0}(\cos \phi)= & 1, \\
\mathcal{T}_{1}(\cos \phi)= & (\cos \phi)\left(1+\mu_{2}\right)^{-1 / 2}, \\
\mathcal{T}_{2}(\cos \phi)= & \left(\cos 2 \phi-\mu_{2}\right)\left(1-2 \mu_{2}^{2}+\mu_{4}\right)^{-1 / 2}, \\
\mathcal{T}_{3}(\cos \phi)= & \left(\cos 3 \phi-\frac{\mu_{2}+\mu_{4}}{1+\mu_{2}} \cos \phi\right)\left(1+\mu_{6}-\frac{\left(\mu_{2}+\mu_{4}\right)^{2}}{1+\mu_{2}}\right)^{-1 / 2}, \\
\mathcal{T}_{4}(\cos \phi)= & \left(\cos 4 \phi-\frac{\mu_{2}-2 \mu_{2} \mu_{4}+\mu_{6}}{1-2 \mu_{2}^{2}+\mu_{4}} \cos 2 \phi+\frac{\mu_{2}\left(\mu_{2}+\mu_{6}\right)-\mu_{4}\left(1+\mu_{4}\right)}{1-2 \mu_{2}^{2}+\mu_{4}}\right) \\
& \quad \times\left(1-2 \mu_{4}^{2}+\mu_{8}-\frac{\left(\mu_{2}-2 \mu_{2} \mu_{4}+\mu_{6}\right)^{2}}{1-2 \mu_{2}^{2}+\mu_{4}}\right)^{-1 / 2},
\end{aligned}
$$


and

$\mathcal{V}_{0}(\cos \phi)=0$

$\mathcal{V}_{1}(\cos \phi)=(\sin \phi)\left(1-\mu_{2}\right)^{-1 / 2}$,

$\mathcal{V}_{2}(\cos \phi)=(\sin 2 \phi)\left(1-\mu_{4}\right)^{-1 / 2}$,

$\mathcal{V}_{3}(\cos \phi)=\left(\sin 3 \phi-\frac{\mu_{2}-\mu_{4}}{1-\mu_{2}} \sin \phi\right)\left(1-\mu_{6}-\frac{\left(\mu_{2}-\mu_{4}\right)^{2}}{1-\mu_{2}}\right)^{-1 / 2}$,

$\mathcal{V}_{4}(\cos \phi)=\left(\sin 4 \phi-\frac{\mu_{2}-\mu_{6}}{1-\mu_{4}} \sin 2 \phi\right)\left(1-\mu_{8}-\frac{\left(\mu_{2}-\mu_{6}\right)^{2}}{1-\mu_{4}}\right)^{-1 / 2}$.

\section{References}

[1] de Gennes P G 1974 The Physics of Liquid Crystals (Oxford: Clarendon)

[2] Derjaguin B V, Altoiz B A and Nikitenko J J 1991 J. Colloid Interface Sci. 145441

[3] Bogoliubov N N 1971 Selected Papers vol 3 (Kiev: Naukova Dumka) p 174 (in Russian)

[4] Sokolovska T G, Sokolovskii R O and Holovko M F 2000 Phys. Rev. E 626771

[5] Sokolovska T G, Sokolovskii R O and Holovko M F 2001 Phys. Rev. E 64051710

[6] Maier W and Saupe A 1959 Z. Naturf. A 14882

[7] Lebwohl P A and Lasher G 1972 Phys. Rev. A 6426

[8] Hansen J-P and McDonald I R 1986 Theory of Simple Liquids (London: Academic)

[9] Henderson D 1992 Fundamentals of Inhomogeneous Fluids ed D Henderson (New York: Dekker) chapter 4

[10] Lovett R A, Mou C Y and Buff F P 1976 J. Chem. Phys. 65570

[11] Wertheim M 1976 J. Chem. Phys. 652377

[12] Lado F, Lomba E and Martín C 2004 J. Mol. Liq. 11251

[13] Kunz H and Zumbach G 1992 Phys. Rev. B 46662

[14] Lado F 1973 Phys. Rev. A 82548

[15] Lado F and Lomba E 1998 Phys. Rev. Lett. 803535

[16] Lado F, Lomba E and Weis J J 1998 Phys. Rev. E 583478

[17] Lomba E, Lado F and Weis J J 2000 Phys. Rev. E 613838

[18] Fariñas-Sanchez A I, Paredes R and Berche B 2003 Phys. Lett. A 308461

[19] Berezinskii V L 1971 Sov. Phys. -JETP 32493

[20] Kosterlitz J M and Thouless D J 1973 J. Phys. C: Solid State Phys. 61181

[21] Arfken G 1985 Mathematical Methods for Physicists (New York: Academic)

[22] Lado F 1968 J. Chem. Phys. 493092

[23] Lado F 1971 Comput. Phys. 8417

[24] Press W H and Teukolsky S A 1990 Comput. Phys. 4423

[25] Swendsen R H and Wang J S 1987 Phys. Rev. Lett. 5886

[26] Lomba E, Martín C and Almarza N G 2003 Mol. Phys. 1011667

[27] Lomba E, Martín C, Almarza N G and Lado F 2005 Phys. Rev. E at press

[28] Saupe A 1964 Z. Naturf. A 19161

[29] Eppenga R and Frenkel D 1984 Mol. Phys. 521303

[30] Chen X S, Kasch M and Forstmann F 1991 Phys. Rev. Lett. 672674

[31] Chen X S, Kasch M and Forstmann F 1992 Phys. Rev. Lett. 69694

[32] Sokolovska T G, Holovko M F and Sokolovskii R O 1997 Ukr. Fiz. Zh. 421304 


\section{Queries for IOP paper 193055}

\section{Journal: JPhysCM}

Author: $\quad$ F Lado et al

Short title: A planar nematogenic liquid in crossed external fields

Page 15

Query 1:-

Author: Figures A1-A5 and tables A1 and A2 have been changed to figures 1-5 and tables 1 and 2 respectively, since it is difficult to place these in the appendix, also since these are mentioned in the main text (and not referred to in the appendix). Please check whether the placement of the figures and tables is OK.

Page 24

Query 2:-

Author: [27]: Any update? 